\title{
On Performance of Vector OFDM With Linear Receivers
}

\author{
Yabo Li, Member, IEEE, Ibo Ngebani, Xiang-Gen Xia, Fellow, IEEE, and \\ Anders Høst-Madsen, Senior Member, IEEE
}

\begin{abstract}
Vector Orthogonal Frequency Division Multiplexing (OFDM) for single transmit antenna systems is a general transmission scheme, where OFDM and Single-Carrier Frequency Domain Equalization (SC-FDE) can be treated as two special/extreme cases. Due to its flexibility, it has drawn more and more attention recently. So far, all the studies about Vector OFDM assume the Maximum Likelihood (ML) receiver. In this paper, we investigate the performance of Vector OFDM with linear receivers, i.e., the Zero-Forcing (ZF) and Minimum Mean Square Error (MMSE) receivers. We first show that the detection SNR gap between the $M M S E$ and $Z F$ receivers increases with both channel SNR and the vector blocks (VB) size defined in Vector OFDM. Then, it is proved that for both $\mathrm{ZF}$ and MMSE receivers, all the transmitted symbols have equal performance. This is different from the Vector OFDM with ML receiver, where different VBs may have different coding gain, and thus may have different performances. We analyze the diversity order for Vector OFDM with MMSE receiver, and show that, regardless of the Vector OFDM symbol length $N$, the diversity order can be represented as $\min \left\{\left\lfloor M 2^{-R}\right\rfloor, D\right\}+1$, where $M$ is the VB size, $R$ is the spectrum efficiency in bits/symbol, and $D$ is the maximum delay of the multipath channel. For Vector OFDM with $\mathrm{ZF}$ receiver, we show that the diversity order equals 1 and the performance is the same as the conventional OFDM at high SNR.
\end{abstract}

Index Terms-Diversity order, linear receiver, MMSE, multipath diversity, OFDM, SC-FDE, signal space diversity, vector OFDM, ZF.

\section{INTRODUCTION}

$\mathbf{O}$ RTHOGONAL FREQUENCY DIVISION MULTIPLEXING (OFDM) [1], [2], as a low complexity transmission scheme in multipath channels, has been widely adopted in the next generation wireless communication systems

Manuscript received February 20, 2012; revised June 05, 2012; accepted June 05, 2012. Date of publication June 21, 2012; date of current version September 11,2012 . The associate editor coordinating the review of this manuscript and approving it for publication was Dr. Tong Zhang. The work of X.-G. Xia was supported in part by the National Science Foundation (NSF) by Grant CCF0964500 and the World Class University (WCU) Program, National Research Foundation, Korea. The work of A. Høst-Madsen was supported in part by the NSF Grant CCF 1017823. This work was supported by the National Science Foundation of China (NSFC) by Grant 61010197 , by the Chinese Ministry of Education New Faculty Fund by Grant 20100101120039, and by the National Science and Technology Major Project by Grant 2011ZX03003-003-03.

Y. Li and I. Ngebani are with the Department of Information Science and Electrical Engineering, Zhejiang Provincial Key Laboratory of Information Network Technology, Zhejiang University, Hangzhou, Zhejiang, P. R. China (e-mail: yaboli@zju.edu.cn; iboz5@yahoo.com).

X.-G. Xia is with the Department of Electrical and Computer Engineering, University of Delaware, Newark, DE 19716 USA. He is also with Chonbuk National University, Jeonju, South Korea (e-mail: xxia@ee.udel.edu).

A. Høst-Madsen is with the Department of Electrical Engineering, University of Hawaii at Manoa, Honolulu, HI 96822 USA (e-mail: ahm@hawaii.edu).

Color versions of one or more of the figures in this paper are available online at http://ieeexplore.ieee.org.

Digital Object Identifier 10.1109/TSP.2012.2205573 such as WiMAX [3], LTE [4], and WiFi [5]. Although OFDM has low complexity, without coding, it cannot exploit the multipath diversity, and therefore performs worse than single carrier transmission with time domain equalizers. Furthermore, OFDM has very high Peak-to-Average Power Ratio (PAPR), which puts high requirements on the Power Amplifier (PA) and increases the transceiver cost [6]. To mitigate PAPR while retaining low complexity, Single-Carrier Frequency Domain Equalization (SC-FDE) [7]-[10] was proposed. At the transmitter, SC-FDE removes the Inverse Fast Fourier Transform (IFFT) block of OFDM, so the PAPR is reduced. At the receiver, SC-FDE first does FFT and channel equalization in the frequency domain, and then does IFFT and demodulation/detection in the time domain. Compared to OFDM, it can be seen that SC-FDE has unbalanced transmitter and receiver complexities. It is further shown that for high order signal constellations, such as 64QAM, SC-FDE suffers performance loss [11]. Therefore, it is commonly agreed that OFDM is more suitable for high data rate and high cost transceivers, while SC-FDE is more suitable for low data rate and low cost transceivers. For example, in LTE [4], OFDM/OFDMA (Orthogonal Frequency Division Multiple Access) is used in the downlink, where high data rate is more important, and SC-FDE/SC-FDMA (Single-Carrier Frequency Division Multiple Access) is used in the uplink, where low cost is more important. In the next generation millimeter-wave based WiFi, i.e., IEEE 802.11ad [5], both OFDM based PHY (Physical Layer) and SC-FDE based PHY are defined, where the OFDM based PHY is for high data rate devices, and the used constellation can be up to 64QAM, while the SC-FDE based PHY is for low data rate, low cost and low power devices. The definition of two PHY schemes complicates the system design.

Vector OFDM (V-OFDM), first proposed by $\mathrm{Xia}$ [12] to reduce the cyclic prefix (CP) overhead and the IFFT size of a single transmit antenna OFDM system, can be treated as a general transmission scheme, where OFDM and SC-FDE are just two special/extreme cases. So, V-OFDM bridges the gap between OFDM and SC-FDE. By adjusting parameters, the V-OFDM based system can be adapted to cater to different system design requirements, such as PAPR, transceiver cost, data rate, performance, etc. Thus, compared with the two PHY schemes in IEEE 802.11ad [5], V-OFDM is an attractive alternative. With regard to different system design aspects of V-OFDM, [13] analyzed the synchronization and guard band settings, [15] introduced the vector channel allocation, [14] exploited the turbo principle to do iterative demodulation and decoding, [16] observed that different vector blocks (VB) may have different performances when the ML (Maximum Likelihood) receiver is used and further proposed a constellation 
rotation scheme to ensure consistent performance for different VBs, [17] thoroughly investigated the V-OFDM performance under multipath Rayleigh fading with the ML receiver.

So far, all the discussions about V-OFDM, such as [12]-[17], are based on the ML receiver, whose complexity increases exponentially with the size of the VB defined in V-OFDM, though the performance increases with the size of the VB as well. It is still unknown how V-OFDM performs with linear receivers, i.e., the Zero Forcing (ZF) and Minimum Mean Square Error (MMSE) receivers. As a special/extreme case of V-OFDM, there are several papers that discussed linear receivers for SC-FDE, see for example [18], [19] . V-OFDM has a more general structure, and thereby involves more parameters. Also, there are several papers that discussed linear receivers for zero-padded (ZP) or CP block transmissions, see for example [19]-[23], where it is shown that the ZP-based transmission can achieve higher diversity gain than the CP-based transmission. V-OFDM is a kind of CP-based block transmission. For Multiple-Input Multiple-Output (MIMO) systems, [24]-[32] discussed the performance of linear receivers. The signal model of V-OFDM has similar form as MIMO, however, there are some key differences. One is that V-OFDM is a single transmit antenna based scheme, the other is that the equivalent channel matrix for V-OFDM has a very special structure, which is quite different from that used in the analysis of MIMO. So, the analysis of the linear receivers for MIMO cannot be applied to V-OFDM in a straightforward manner.

In this paper, we thoroughly investigate the performance of V-OFDM with linear receivers. In the following, we call the V-OFDM with ML, ZF and MMSE receivers ML-V-OFDM, ZF-V-OFDM and MMSE-V-OFDM, respectively. The contributions of this paper can be summarized as follows.

- It is shown that both ZF-V-OFDM and MMSE-V-OFDM do not require explicit matrix inversion and thus have very low complexity, which, different from ML-V-OFDM, does not increase with the VB size defined in V-OFDM.

- It is shown that the gap between the detection SNRs of ZF-V-OFDM and MMSE-V-OFDM increases with both VB size $M$ and channel SNR $\rho$.

- Different from ML-V-OFDM, where different VBs may have different performances [16], [17], we show that for both ZF-V-OFDM and MMSE-V-OFDM, all the VBs have equal performance.

- By using the techniques first proposed by Tajer and Nosratinia [18], we analyze the diversity order of MMSE-VOFDM and show that, regardless of the V-OFDM symbol size $N$, the diversity order, defined as the slope of the SER (Symbol Error Rate) versus SNR curve in the log-log domain, equals $\min \left\{\left\lfloor M 2^{-R}\right\rfloor, D\right\}+1$, where $M$ is the VB size, $R$ is the spectrum efficiency in bits/symbol, and $D$ is the maximum delay of the multipath channel.

- We show that ZF-V-OFDM does not have any diversity gain (i.e., the diversity order equals 1 ) and its performance is the same as the conventional OFDM at high SNR.

The remainder of the paper is organized as follows. In Section II, the system model of V-OFDM is introduced. In Section III, different detection algorithms are introduced and the characteristics of the detection SNRs are analyzed.
In Section IV, the equal performance of different VBs for MMSE-V-OFDM and ZF-V-OFDM is shown. In Section V, the diversity orders of MMSE-V-OFDM and ZF-V-OFDM are analyzed. In Section VI, some numerical results are provided to validate the theoretical analysis.

\section{Vector OFDM System Model}

V-OFDM was first proposed in [12] for single transmit antenna systems. It is a generalization of OFDM, and is able to combat spectrum nulls in the channel and collect multipath diversity. In the following we first introduce the received signal model of V-OFDM, and then show that the conventional OFDM and SC-FDE are just two special/extreme cases of V-OFDM.

\section{A. The Received Signal Model}

Similar to conventional OFDM, in V-OFDM, the modulated symbols are processed block-by-block. Assume that there are $N=L M$ modulated symbols in one block, and denote them as $\left\{x_{n}\right\}_{n=0}^{N-1}$. Different from conventional OFDM, V-OFDM further divides the length $N$ block into $L$ VBs, where each VB has size $M$. Denote the $l$ th $\mathrm{VB}$ as

$$
\mathbf{x}_{l}=\left[x_{l M}, x_{l M+1} \ldots, x_{l M+M-1}\right]^{T}, \quad l=0,1, \ldots, L-1 .
$$

We call $\mathrm{x}_{l}$ the $l$ th transmit VB. Instead of doing IFFT of size $\mathrm{N}$ as in conventional OFDM, V-OFDM does component-wise vector IFFT of size $L$ over the VBs, i.e., calculates

$$
\overline{\mathbf{x}}_{q}=\frac{1}{L} \sum_{l=0}^{L-1} \mathbf{x}_{l} e^{j \frac{2 \pi q l}{L}}, \quad q=0,1, \ldots, L-1 .
$$

$\overline{\mathbf{x}}_{q}$ is a column vector of size $M$ and is denoted as

$$
\overline{\mathbf{x}}_{q}=\left[\bar{x}_{q M}, \bar{x}_{q M+1}, \ldots, \bar{x}_{q M+M-1}\right]^{T} .
$$

Rewrite the vectors $\left\{\overline{\mathbf{x}}_{q}\right\}_{q=0}^{L-1}$, as a size $N$ row vector, which is

$$
\left[\bar{x}_{0}, \bar{x}_{1}, \ldots, \bar{x}_{N-1}\right]=\left[\overline{\mathbf{x}}_{0}^{T}, \overline{\mathbf{x}}_{1}^{T}, \ldots, \overline{\mathbf{x}}_{L-1}^{T}\right] .
$$

As in conventional OFDM, CP is added to this size- $N$ row vector. Assuming that the length of $\mathrm{CP}$ is $P$, to avoid the interblock-interference, $P$ should satisfy $P>D$, where $D$ is the maximum delay of the multipath channel. Without loss of generality, we assume that $P$ is a multiple of the VB size $M$, i.e., $P=K M$. Then, after adding CP, the transmitted symbol sequence in the time domain can be written as ${ }^{1}$

$$
\left[\overline{\mathbf{x}}_{L-K}^{T}, \overline{\mathbf{x}}_{L-K+1}^{T}, \ldots, \overline{\mathbf{x}}_{L-1}^{T}, \overline{\mathbf{x}}_{0}^{T}, \overline{\mathbf{x}}_{1}^{T}, \ldots, \overline{\mathbf{x}}_{L-1}^{T}\right] .
$$

At the receiver, after removing $\mathrm{CP}$, the received signal is the circular convolution of the transmitted signal and the CIR (Channel Impulse Response), which can be written as

$$
\bar{y}_{n}=\sum_{d=0}^{D} h_{d} \bar{x}_{(n-d)_{N}}+w_{n}, \quad n=0,1, \ldots, N-1,
$$

where $\left\{h_{d}\right\}_{d=0}^{D}$ is the CIR, $w_{n} \sim \mathcal{C} \mathcal{N}\left(0, \sigma^{2}\right)$ is the AWGN, and $(n)_{N}$ means $n \bmod N$. The receiver then divides the length

${ }^{1}$ For large $M$, the CP length $P$ may be smaller than $M$. In this case, we just move part of the symbols in the last VB to the front as the $\mathrm{CP}$, and the notation of the transmitted symbol may be different from the (1). 
$N$ block $\left[\bar{y}_{0}, \bar{y}_{1}, \ldots, \bar{y}_{N-1}\right]$ into $L$ size $M$ column vectors $\left\{\overline{\mathbf{y}}_{q}\right\}_{q=0}^{L-1}$, where $\overline{\mathbf{y}}_{q}$ is

$$
\overline{\mathbf{y}}_{q}=\left[\bar{y}_{q M}, \bar{y}_{q M+1}, \ldots, \bar{y}_{q M+M-1}\right]^{T} .
$$

Taking component-wise vector FFT of size $L$, we have

$$
\mathbf{y}_{l}=\sum_{q=0}^{L-1} \overline{\mathbf{y}}_{q} e^{-j \frac{2 \pi q l}{L}}, \quad l=0,1, \ldots, L-1 .
$$

Write the length $M$ column vector $\mathbf{y}_{l}$ as

$$
\mathbf{y}_{l}=\left[y_{l M}, y_{l M+1}, \ldots, y_{l M+M-1}\right]^{T} .
$$

We call $\mathbf{y}_{l}$ the $l$ th receive VB.

Denote

$$
H_{k}=\sum_{d=0}^{D} h_{d} e^{-j \frac{2 \pi k d}{N}}, \quad k=0,1, \ldots, N-1
$$

i.e., the frequency domain channel coefficient at the $k$ th subcarrier in conventional OFDM with size $N$ FFT/IFFT. Define the $M \times M$ diagonal matrix $\overline{\mathbf{H}}_{l}$ as

$$
\overline{\mathbf{H}}_{l}=\operatorname{diag}\left\{H_{l}, H_{l+L}, \ldots, H_{l+(M-1) L}\right\} .
$$

Assuming perfect synchronization, with some signal processing manipulations [12], [13], the transmit $\mathrm{VB} \mathrm{x}_{l}$ and the receive $\mathrm{VB} \mathbf{y}_{l}$ have the relation

$$
\mathbf{y}_{l}=\mathbf{H}_{l} \mathbf{x}_{l}+\mathbf{w}_{l}, \quad l=0,1, \ldots, L-1
$$

where $\mathbf{w}_{l}=\left[w_{l, 0}, w_{l, 1}, \ldots, w_{l, M-1}\right]^{T}$ is the noise vector, whose entries are i.i.d. and $\mathcal{C N}\left(0, \sigma^{2}\right)$ distributed, and the equivalent channel matrix $\mathbf{H}_{l}$ can be expressed as

$$
\mathbf{H}_{l} \triangleq \mathbf{U}_{l}^{H} \overline{\mathbf{H}}_{l} \mathbf{U}_{l}
$$

where $\mathbf{U}_{l}$ is a unitary matrix, whose entry in the $s$ th row $(s=$ $0,1, \ldots, M-1)$ and $m$ th $(m=0,1, \ldots, M-1)$ column equals

$$
\left[\mathbf{U}_{l}\right]_{s, m}=\frac{1}{\sqrt{M}} \exp \left(-j \frac{2 \pi(l+s L) m}{N}\right) .
$$

It is not difficult to verify that $\mathbf{U}_{l}$ can be written as a product of a DFT matrix and a diagonal matrix, i.e.,

$$
\mathbf{U}_{l}=\mathbf{F}_{M} \boldsymbol{\Lambda}_{l}
$$

where $\mathbf{F}_{M}$ is the $M \times M$ DFT matrix and $\boldsymbol{\Lambda}_{l}$ is defined as

$$
\boldsymbol{\Lambda}_{l} \triangleq \operatorname{diag}\left\{1, e^{\frac{-j 2 \pi l}{N}}, e^{\frac{-j 2 \pi 2 l}{N}}, \ldots, e^{\frac{-j 2 \pi(M-1) l}{N}}\right\} .
$$

Assuming that $\mathbb{E}\left\{\left|x_{n}\right|^{2}\right\}=1, n=0,1, \ldots, N-1$, the transmit SNR can be defined as $\rho \triangleq \frac{1}{\sigma^{2}}$.

Note that although the CP overhead of the V-OFDM above is not changed, the IFFT size is reduced from $N$ to $L$ by $M$ times. This IFFT size reduction also reduces the PAPR.

\section{B. V-OFDM and Other Modulation Schemes}

As introduced before, V-OFDM is a general modulation scheme, and conventional OFDM and SC-FDE are two special/extreme cases of V-OFDM.

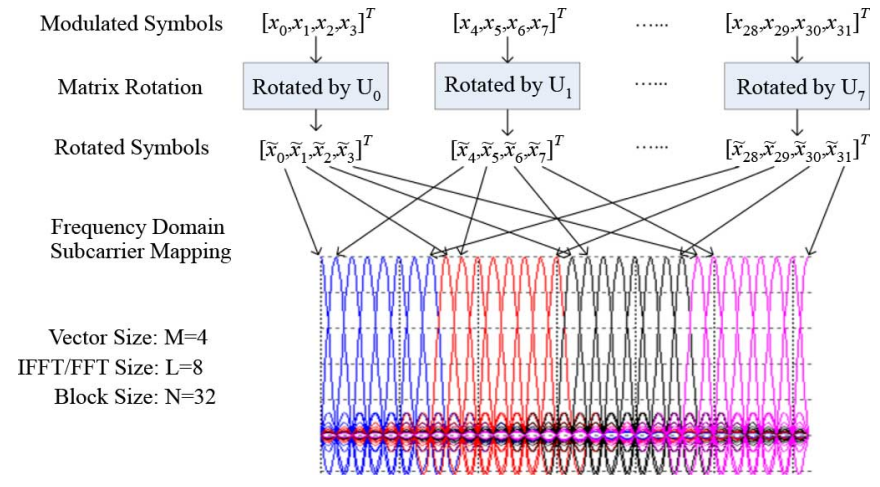

Fig. 1. V-OFDM modulation and mapping.

If we choose parameter $M=1$, then $L=N$ and the received signal model (4) has a simple scalar form

$$
y_{l}=H_{l} x_{l}+w_{l}, \quad l=0,1, \ldots, N-1
$$

which is exactly conventional OFDM.

If we choose parameter $M=N$, then $L=1, \boldsymbol{\Lambda}_{l}=\mathbf{I}$, and $\mathbf{U}_{l}=\mathbf{F}_{N}$. In this case, in (4), $\mathbf{U}_{l}^{H} \overline{\mathbf{H}} \mathbf{U}_{l}$ can be treated as the singular value decomposition of the equivalent channel $\mathbf{H}_{l}$, where $\mathbf{H}_{l}$ has a circulant structure, which equals

$$
\mathbf{H}_{l}=\left[\begin{array}{ccccccc}
h_{0} & 0 & \cdots & h_{D} & \cdots & h_{2} & h_{1} \\
h_{1} & h_{0} & 0 & \cdots & h_{D} & \cdots & h_{2} \\
\vdots & \ddots & \ddots & \ddots & \ddots & \ddots & \vdots \\
0 & \cdots & h_{D} & \cdots & h_{1} & h_{0} & 0 \\
0 & \cdots & 0 & h_{D} & \cdots & h_{1} & h_{0}
\end{array}\right]_{N \times N}
$$

In this case, the signal model (4) is exactly SC-FDE.

When $L \neq N$ and $M \neq N$, V-OFDM can be treated as an implementation of signal space diversity (or modulation diversity) [14], [33] [34]. If we write the received signal model (4) as

$$
\mathbf{U}_{l} \mathbf{y}_{l}=\overline{\mathbf{H}}_{l} \mathbf{U}_{l} \mathbf{x}_{l}+\mathbf{U}_{l} \mathbf{w}_{l},
$$

then the unitary matrix $\mathbf{U}_{l}$ can be treated as a rotation matrix, the same as that defined in the signal space diversity [33], [34] . Fig. 1 shows the equivalent transmit processing of V-OFDM. In Fig. 1, the parameters used are: $M=4, L=8$, and $N=$ 32. After matrix rotation, the rotated symbols are mapped to $M$ subcarriers that are evenly distributed in the frequency band.

\section{Detection Algorithms}

In this section, we derive different detection algorithms for V-OFDM, assuming that the channel is perfectly known by the receiver.

\section{A. ML Detection}

Based on (4), the ML detection can be written as

$$
\hat{\mathbf{x}}_{l}^{M L}=\arg \min _{\mathbf{x}_{l}}\left\|\mathbf{y}_{l}-\mathbf{H}_{l} \mathbf{x}_{l}\right\|^{2}, \quad l=0,1, \ldots, L-1 .
$$

Clearly, complexity of ML-V-OFDM grows exponentially with the VB size $M$ and the modulation order. In [16], the authors observed that different VBs may have different performances and further proposed a constellation rotation scheme to ensure 
consistent performance over different VBs. In [12], [17], the authors analyzed the performance of ML-V-OFDM, and in [17], it is shown that the majority of the VBs can achieve the maximum diversity order, which equals $\min \{M, D+1\}$.

\section{B. ZF Detection}

The equivalent channel matrix $\mathbf{H}_{l}$ in (4) is the multiplication of two unitary matrices and a diagonal matrix, so its inversion is simple to calculate, which is

$$
\begin{aligned}
\mathbf{C}_{l}^{Z F} & \triangleq \mathbf{H}_{l}^{-1}=\mathbf{U}_{l}^{H} \overline{\mathbf{H}}_{l}^{-1} \mathbf{U}_{l} \\
& =\mathbf{U}_{l}^{H} \operatorname{diag}\left\{\frac{1}{H_{l}}, \frac{1}{H_{l+L}}, \ldots, \frac{1}{H_{l+(M-1) L}}\right\} \mathbf{U}_{l} .
\end{aligned}
$$

The signal after the ZF processing is

$$
\begin{aligned}
\hat{\mathbf{y}}_{l}^{Z F} & =\mathbf{C}_{l}^{Z F} \mathbf{y}_{l}=\mathbf{x}_{l}+\mathbf{C}_{l}^{Z F} \mathbf{w}_{l} \\
& \triangleq\left[\hat{y}_{l M}^{Z F}, \hat{y}_{l M+1}^{Z F}, \ldots, \hat{y}_{l M+M-1}^{Z F}\right]^{T}
\end{aligned}
$$

and the symbol-by-symbol detection is

$$
\hat{x}_{n}^{Z F}=\arg \min _{x_{n}}\left|\hat{y}_{n}^{Z F}-x_{n}\right|^{2}, \quad n=0,1, \ldots, N-1 .
$$

The noise covariance of the signal after ZF processing can be calculated as

$$
\begin{aligned}
\mathbf{R}_{w}^{Z F}= & \mathbb{E}\left[\mathbf{C}_{l}^{Z F} \mathbf{w}_{l} \mathbf{w}_{l}^{H}\left(\mathbf{C}_{l}^{Z F}\right)^{H}\right] \\
= & \mathbf{U}_{l}^{H} \operatorname{diag}\left\{\frac{\sigma^{2}}{\left|H_{l}\right|^{2}}, \frac{\sigma^{2}}{\left|H_{l+L}\right|^{2}}, \ldots,\right. \\
& \left.\frac{\sigma^{2}}{\left|H_{l+(M-1) L}\right|^{2}}\right\} \mathbf{U}_{l} .
\end{aligned}
$$

Because $\mathbf{U}_{l}=\mathbf{F}_{M} \boldsymbol{\Lambda}_{l}$, where $\mathbf{F}_{M}$ is a DFT matrix and $\boldsymbol{\Lambda}_{l}$ is a diagonal matrix defined in (5), it is not difficult to see that the diagonal elements in $\mathbf{R}_{w}^{Z F}$ are equal, and can be written as

$$
\left[\mathbf{R}_{w}^{Z F}\right]_{m, m}=\frac{1}{M} \sum_{n=0}^{M-1} \frac{\sigma^{2}}{\left|H_{l+n L}\right|^{2}}, \quad m=0,1, \ldots, M-1 .
$$

Since the ZF estimation is unbiased, and $\mathbb{E}\left[x_{n}\right]=1$, for $n=$ $0,1, \ldots, N-1$, we have that the detection SNR for the $m$ th element in the $l$ th $\mathrm{VB} \mathbf{x}_{l}$ is

$$
\rho_{l, m}^{Z F} \triangleq \frac{\mathbb{E}\left[x_{l M+m}\right]}{\left[\mathbf{R}_{w}^{Z F}\right]_{m, m}}=\left[\frac{1}{M} \sum_{k=0}^{M-1} \frac{1}{\rho\left|H_{l+k L}\right|^{2}}\right]^{-1}
$$

and $\rho_{l, 0}^{Z F}=\rho_{l, 1}^{Z F}=\cdots=\rho_{l, M-1}^{Z F} \triangleq \rho_{l}^{Z F}$.

ZF-V-OFDM has a simple matrix inversion and simple symbol-by-symbol detection. Its complexity does not increase with the VB size $M$, and is much lower than that of ML-V-OFDM.

\section{MMSE Detection}

Assuming that both channel coefficients and noise variance are known by the receiver, we can use MMSE detection to fur- ther improve the performance. The weight matrix of MMSE can be calculated as

$$
\begin{aligned}
\mathbf{C}_{l}^{M M S E} \triangleq\left(\mathbf{H}_{l}^{H} \mathbf{H}_{l}+\rho^{-1} \mathbf{I}\right)^{-1} \mathbf{H}_{l}^{H} & \\
= & \mathbf{U}_{l}^{H} \operatorname{diag}\left\{\frac{H_{l}^{*}}{\left|H_{l}\right|^{2}+\rho^{-1}}, \ldots,\right. \\
& \left.\frac{H_{l+(M-1) L}^{*}}{\left|H_{l+(M-1) L}^{2}\right|+\rho^{-1}}\right\} \mathbf{U}_{l}
\end{aligned}
$$

where the second equation is also due to the special structure of $\mathbf{H}_{l}$. Compared with ZF-V-OFDM, it can be seen that MMSE-VOFDM does not need explicit matrix inversion as well and its complexity is the same as that of ZF-V-OFDM, which does not increase with $M$. After MMSE filtering, we have

$$
\begin{aligned}
\hat{\mathbf{y}}_{l}^{M M S E}= & \mathbf{C}_{l}^{M M S E} \mathbf{y}_{l} \\
= & \mathbf{U}_{l}^{H} \operatorname{diag}\left\{\frac{\left|H_{l}\right|^{2}}{\left|H_{l}\right|^{2}+\rho^{-1}}, \ldots,\right. \\
& \left.\frac{\left|H_{l+(M-1) L}\right|^{2}}{\left|H_{l+(M-1) L}^{2}\right|+\rho^{-1}}\right\} \mathbf{U}_{l} \mathbf{x} \\
& \quad+\mathbf{C}_{l}^{M M S E} \mathbf{w} \\
\triangleq & {\left[\hat{y}_{l M}^{M M S E}, \hat{y}_{l M+1}^{M M S E}, \ldots, \hat{y}_{l M+M-1}^{M M S E}\right]^{T} }
\end{aligned}
$$

and the $m$ th element of $\hat{\mathbf{y}}_{l}^{M M S E}$ can be written as

$$
\hat{y}_{l M+m}^{M M S E}=C_{l M+m} x_{l M+m}+n_{l M+m},
$$

where

$$
C_{l M+m}=\frac{1}{M} \sum_{m=0}^{M-1} \frac{\left|H_{l+m L}\right|^{2}}{\left|H_{l+m L}\right|^{2}+\rho^{-1}}
$$

is the scale factor of the symbol $x_{l M+m}$, and

$$
\begin{array}{r}
n_{l M+m}=\sum_{j=0, j \neq m}^{M} x_{l M+j} \sum_{k=0}^{M-1} u_{l, k, m}^{*} u_{l, k, j} \\
\times \frac{\left|H_{l+k L}\right|^{2}}{\left|H_{l+k L}\right|^{2}+\rho^{-1}}+\hat{w}_{m}
\end{array}
$$

is the interference plus noise, where $u_{l, i, j}=\left[\mathbf{U}_{l}\right]_{i, j}$ and $\hat{w}_{m}=$ $\left[\mathbf{C}_{l}^{M M S E} \mathbf{w}\right]_{m}$.

Then, the symbol-by-symbol detection is

$$
\hat{x}_{n}^{M M S E}=\arg \min _{x_{n}}\left|\hat{y}_{n}^{M M S E}-C_{n} x_{n}\right|^{2}
$$

and the detection SNR or Signal to Interference plus Noise Ratio (SINR) for the $m$ th element in the $l$ th $V B \mathbf{x}_{l}$ is (see Appendix A for detailed derivations)

$\rho_{l, m}^{M M S E} \triangleq \frac{\left|C_{l M+m}\right|^{2}}{\mathbb{E}\left[\left|n_{l M+m}\right|^{2}\right]}=\left[\frac{1}{M} \sum_{k=0}^{M-1} \frac{1}{\rho\left|H_{l+k L}\right|^{2}+1}\right]^{-1}-1$

and $\rho_{l, 0}^{M M S E}=\rho_{l, 1}^{M M S E}=\cdots=\rho_{l, M-1}^{M M S E} \triangleq \rho_{l}^{M M S E}$.

Based on these analyses, we have that, for both ZF-V-OFDM and MMSE-V-OFDM, different elements in the same VB have the same detection SNR. 


\section{The Relationship Between $\rho_{l}^{z f}$ and $\rho_{l}^{m m s e}$}

Based on (8) and (11) we have the following theorem.

Theorem 1: Denote the $m$ th column of $\mathbf{H}_{l}$ as $\mathbf{h}_{l, m}$ and the matrix of $\mathbf{H}_{l}$ after deleting the $m$ th column as $\mathbf{H}_{l, m}$, which is an $M \times(M-1)$ matrix. When $\rho \rightarrow \infty$, the gap between the detection SNRs of the ZF-V-OFDM and the MMSE-V-OFDM can be written as

$$
\lim _{\rho \rightarrow \infty}\left(\rho_{l}^{M M S E}-\rho_{l}^{Z F}\right)=\left\|\mathbf{h}_{l}^{H} \mathbf{H}_{l, m}\left(\mathbf{H}_{l, m}^{H} \mathbf{H}_{l, m}\right)^{-1}\right\|^{2}
$$

which is independent of $m$. As $\rho \rightarrow \infty$, the ratio between $\rho_{l}^{M M S E}$ and $\rho_{l}^{Z F}$ approaches 1, i.e.,

$$
\lim _{\rho \rightarrow \infty} \frac{\rho_{l}^{M M S E}}{\rho_{l}^{Z F}}=1 .
$$

Proof: See Appendix B

Compared with the linear receiver for MIMO, several points to notice.

- For ZF-V-OFDM and MMSE-V-OFDM, the detection SNRs $\rho_{l, m}^{Z F}$ and $\rho_{l, m}^{M M S E}$ are independent of $m$. This is different from the MIMO with linear receivers, where different MIMO streams may have different detection SNRs. The left-hand side of (12) is independent of $m$, which means that the right hand side of (12) is also independent of $\mathrm{m}$, which can be, in fact, directly proved by using the special structure of $\mathbf{H}_{l}$.

- In [28] and [30], the authors show that for MIMO, the gap between the MMSE detection SNR (denoted as $\rho_{m}^{M M S E}$ ) and the ZF detection SNR (denoted as $\rho_{m}^{Z F}$ ), i.e., $\rho_{m}^{M M S E}-\rho_{m}^{Z F}$, is independent of $\rho_{m}^{Z F}$, where $m$ means the $m$ th MIMO stream. Further, in [28], explicit closed-form PDF (Probability Density Function) of $\rho_{m}^{M M S E}-\rho_{m}^{Z F}$ for i.i.d. MIMO Rayleigh channels is obtained. In V-OFDM, the channel $\mathbf{H}_{l}$ has a special structure and does not have i.i.d. Rayleigh fading elements, thus $\rho_{m}^{M M S E}-\rho_{m}^{Z F}$ is not independent of $\rho_{m}^{Z F}$ anymore. So, the PDF of $\rho_{m}^{M M S E}-\rho_{m}^{Z F}$ obtained in [28] cannot be applied here.

- Theorem 1 is concerned with the gap of the detection SNRs for one channel implementation. This gap cannot be simply mapped to the SNR gap in SER versus SNR curves, where the SNR there is the channel SNR $\rho$. Thus, Theorem 1 does not mean that the SER versus SNR curves of MMSE and ZF only have a constant SNR shift.

- For ML-V-OFDM, it has been shown that the diversity order increases with the VB size $M[12]$, [16], [17]. For MMSE-V-OFDM and ZF-V-OFDM, when CIR is fixed, the gap $\rho_{l}^{M M S E}-\rho_{l}^{Z F}$ does not have to increase with $M$. However, we can show that, after averaging over the channel implementations, the SNR gap, i.e., the $\mathbb{E}_{\mathbf{H}_{l}}\left[\rho_{l}^{M M S E}-\rho_{l}^{Z F}\right]$, increases with $M$, which means that the larger the $M$ is, the better the performance of MMSE-V-OFDM is compared to that of ZF-V-OFDM. Since the closed-form PDF of $\rho_{l}^{M M S E}-\rho_{l}^{Z F}$ is difficult to obtain for MMSE-V-OFDM, we show this conclusion by simulations in Section VI.

\section{The Performance IndePendence of Vector BLOCK INDEX $l$}

Since ZF-V-OFDM and MMSE-V-OFDM use scalar detectors as in (7) and (10), their performances depend only on the distributions of the detection SNRs, i.e., $\rho_{l}^{Z F}$ and $\rho_{l}^{M M S E}$. According to (8) and (11), we can see that the distributions of the detection SNRs further depend on the joint PDF of the channel coefficients $\left\{H_{l+m L}\right\}_{m=0}^{M-1}$.

Define $\mathbf{h} \triangleq\left[h_{0}, h_{1}, \ldots, h_{D}\right]^{T}$ and $\quad \overline{\mathbf{h}}_{l} \triangleq$ $\left[H_{l}, H_{l+L}, \ldots, H_{l+(M-1) L}\right]^{T}$. Based on (2), $\overline{\mathbf{h}}_{l}$ can be written as

$$
\overline{\mathbf{h}}_{l}=\mathbf{Q}_{l} \mathbf{h}, \quad l=0,1, \ldots, L-1
$$

where $\mathbf{Q}_{l}$ is an $M \times(D+1)$ matrix, whose element in the $m$ th row and $d$ th column equals $\left[\mathbf{Q}_{l}\right]_{m, d}=\frac{1}{\sqrt{M}} \exp \left(-j 2 \pi(l+m L) \frac{d}{N}\right)$, where $m=0,1, \ldots$, $M-1$ and $d=0,1, \ldots, D$.

The following lemma shows that the joint PDF of the elements in $\overline{\mathbf{h}}_{l}$ is independent of $l$.

Lemma 1: Assume that the elements in $\mathbf{h}$ are independent and zero mean complex Gaussian distributed, $\overline{\mathbf{h}}_{l}=\mathbf{Q}_{l} \mathbf{h}$ and $\mathbf{Q}_{l}$ is defined as $\left[\mathbf{Q}_{l}\right]_{m, d}=\frac{1}{\sqrt{M}} \exp \left(-j 2 \pi(l+m L) \frac{d}{N}\right)$, where $l=0,1, \ldots, L-1, m=0,1, \ldots, M-1$ and $d=0,1, \ldots, D$. Then, the joint PDF of the elements in $\overline{\mathbf{h}}_{l}$ is independent of $l$.

Proof: Since $\overline{\mathbf{h}}_{l}$ is a linear combination of $\mathbf{h}$ and the elements in $\mathbf{h}$ are Gaussian distributed, the elements in $\overline{\mathbf{h}}_{l}$ are also Gaussian distributed and the PDF is fully determined by the mean and covariance of $\overline{\mathbf{h}}_{l}$. Because $\mathbf{h}$ is zero mean, so is $\overline{\mathbf{h}}_{l}$. The covariance of $\overline{\mathbf{h}}_{l}$ can be calculated as

$$
\mathbf{R}_{\overline{\mathbf{h}}_{l}} \triangleq \mathbb{E}_{\mathbf{h}}\left[\overline{\mathbf{h}}_{l} \overline{\mathbf{h}}_{l}^{H}\right]=\mathbf{Q}_{l} \mathbb{E}_{\mathbf{h}}\left[\mathbf{h h}^{H}\right] \mathbf{Q}_{l}^{H}=\mathbf{Q}_{l} \mathbf{R}_{\mathbf{h}} \mathbf{Q}_{l}^{H}
$$

where $\mathbf{R}_{\mathbf{h}} \triangleq \mathbb{E}_{\mathbf{h}}\left[\operatorname{diag}\left\{\left|h_{0}\right|^{2},\left|h_{1}\right|^{2}, \ldots,\left|h_{D}\right|^{2}\right\}\right]$. The matrix $\mathrm{Q}_{l}$ can be written as

$$
\mathbf{Q}_{l}=\mathbf{Q}_{0} \tilde{\Lambda}_{l}
$$

where $\tilde{\boldsymbol{\Lambda}}_{l} \triangleq \operatorname{diag}\left\{1, e^{\frac{-j 2 \pi l}{N}}, \ldots, e^{\frac{-j 2 \pi D l}{N}}\right\}$. So, we have

$$
\mathbf{R}_{\overline{\mathbf{h}}_{l}}=\mathbf{Q}_{0} \tilde{\mathbf{\Lambda}}_{l} \mathbf{R}_{\mathbf{h}} \tilde{\mathbf{\Lambda}}_{l}^{H} \mathbf{Q}_{0}^{H} .
$$

It is not difficult to see that $\tilde{\Lambda}_{l} \mathbf{R}_{\mathbf{h}} \tilde{\Lambda}_{l}^{H}=\mathbf{R}_{\mathbf{h}}$ and

$$
\mathbf{R}_{\overline{\mathbf{h}}_{l}}=\mathbf{Q}_{0} \mathbf{R}_{\mathbf{h}}^{H} \mathbf{Q}_{0}^{H}
$$

which means that $\mathbf{R}_{\overline{\mathbf{h}}_{l}}$ is independent of $l$. Since it is Gaussian distributed, we have that the PDF of the elements in $\overline{\mathbf{h}}_{l}$ does not depend on $l$.

According to (8), (11) and Lemma 1, we have the following theorem.

Theorem 2: For ZF-V-OFDM and MMSE-V-OFDM, after averaging over the channel, all the $N$ transmitted symbols $\left\{x_{n}\right\}_{n=0}^{N-1}$ have the same error rate performance.

So, in the following, we focus on the performance analysis of one VB only, without loss of generality, we assume it is $l=0$, and the performance result is valid for all $l=0,1, \ldots, L-1$. 


\section{DiVERSITY ANALYSIS V-OFDM With LINEAR RECEIVERS}

In this section, we use the techniques first proposed by Nosratinia et al. [18], [29] to analyze the diversity order of V-OFDM with linear receivers. We mainly focus on the differences for V-OFDM. When the results in [18], [29] can be applied to V-OFDM directly, we omit the proofs and refer to [18], [29] for details. For completeness, we first define the essentials that are needed for the diversity order analysis.

\section{A. Diversity Analysis}

The SER can be calculated as the pair-wise error probability averaged over the channel and the transmitted symbols, which can be written as

$$
\begin{aligned}
P_{\text {ser }}(R, M, D, N) \triangleq \mathbb{E}_{\mathbf{H}_{l}, x_{n}, x_{m}} & \\
& \times\left\{P\left(x_{n} \rightarrow x_{m} \mid \mathbf{H}_{l}, x_{n} \neq x_{m}\right)\right\} .
\end{aligned}
$$

The SER depends on parameters $R$ (the transmission rate in bits/symbol, or spectrum efficiency), $M$ (the size of VB), $D$ (the channel length), and $N$ (the V-OFDM symbol length). The diversity order is defined as

$$
d(R, M, D, N)=-\lim _{\rho \rightarrow \infty} \frac{\log P_{\text {ser }}(R, M, D, N)}{\log \rho}
$$

We use $d^{Z F}(R, M, D, N)$ and $d^{M M S E}(R, M, D, N)$ to represent the diversity orders of ZF-V-OFDM and MMSE-V-OFDM, respectively.

Following the same approach as in [18], [29], we can define the effective mutual information between the linear filter output $\hat{\mathbf{y}}_{l}$ and the transmitted symbol $\mathbf{x}_{l}$ as

$$
I\left(\hat{\mathbf{y}}_{l} ; \mathbf{x}_{l}\right)=\frac{1}{M} \sum_{m=0}^{M-1} I\left(\hat{y}_{l M+m} ; x_{l M+m}\right)
$$

Due to (8), for ZF-V-OFDM, $I\left(\hat{y}_{l M+m}, x_{l M+m}\right)$ can be written as

$$
\begin{aligned}
I^{Z F} & \left(\hat{y}_{l M+m} ; x_{l M+m}\right) \\
& =\log \left(1+\rho_{l}^{Z F}\right) \\
& =\log \left(1+\left(\frac{1}{M} \sum_{n=0}^{M-1} \frac{1}{\rho\left|H_{l+n L}\right|^{2}}\right)^{-1}\right)
\end{aligned}
$$

and due to (11), for MMSE-V-OFDM, $I\left(\hat{y}_{l M+m}, x_{l M+m}\right)$ can be written as

$$
\begin{aligned}
I^{M M S E}\left(\hat{y}_{l M+m} ; x_{l M+m}\right) \\
\quad=\log \left(1+\rho_{l}^{M M S E}\right) \\
\quad=-\log \left(\frac{1}{M} \sum_{n=0}^{M-1} \frac{1}{\rho\left|H_{l+n L}\right|^{2}+1}\right) .
\end{aligned}
$$

Because the detection SNRs of all the symbols in one VB are the same, the mutual information in (13) equals to the mutual information of each symbol in one VB, i.e.

$$
\begin{aligned}
& I^{Z F}\left(\hat{\mathbf{y}}_{l} ; \mathbf{x}_{l}\right) \\
& \quad=I^{Z F}\left(\hat{y}_{l M+m} ; x_{l M+m}\right) \\
& \quad=\log \left(1+\left(\frac{1}{M} \sum_{n=0}^{M-1} \frac{1}{\rho\left|H_{l+n L}\right|^{2}}\right)^{-1}\right), \\
& I^{M} M S E\left(\hat{\mathbf{y}}_{l} ; \mathbf{x}_{l}\right) \\
& \quad=I^{M M S E}\left(\hat{y}_{l M+m} ; x_{l M+m}\right) \\
& \quad=-\log \left(\frac{1}{M} \sum_{n=0}^{M-1} \frac{1}{\rho\left|H_{l+n L}\right|^{2}+1}\right) .
\end{aligned}
$$

The outage probability is defined as

$$
P_{\text {out }}(R, M, D, N) \triangleq P\left[I\left(\hat{\mathbf{y}}_{l}, \mathbf{x}_{l}\right)<R\right]
$$

which is equal to $P\left[I\left(\hat{y}_{l M+m} ; x_{l M+m}\right)<R\right]$ for ZF-V-OFDM or MMSE-V-OFDM. This means that the above outage probability of the detection of the vector channel (4) after the ZF or the MMSE operator/receiver is indeed that of the scalar channel detection (7) or (10), respectively. Similarly, the outage diversity order is defined as

$$
d_{\text {out }}(R, M, D, N)=-\lim _{\rho \rightarrow \infty} \frac{\log P_{\text {out }}(R, M, D, N)}{\log \rho}
$$

Also, we use $d_{\text {out }}^{Z F}(R, M, D, N)$ and $d_{\text {out }}^{M M S E}(R, M, D, N)$ to represent the outage diversity order for ZF-V-OFDM and MMSE-V-OFDM, respectively.

Similarly as in [18], [29], we define the exponential equal of two functions $f(\rho)$ and $g(\rho)$, write it as $f(\rho) \doteq g(\rho)$, if

$$
\lim _{\rho \rightarrow \infty} \frac{\log f(\rho)}{\log \rho}=\lim _{\rho \rightarrow \infty} \frac{\log g(\rho)}{\log \rho} .
$$

Compared with the analysis of SC-FDE in [18], there are several key differences. One is that in [18], the authors analyzed the diversity order which changes with the spectrum efficiency $R$, the channel length $D$ and the SC-FDE symbol length $N$. Here, for $\mathrm{V}-\mathrm{OFDM}$, besides these parameters, we have the parameter $M$, i.e., the size of VB. The other is that for SC-FDE, it does not make sense to assume that $N<(D+1)$, i.e., the SC-FDE symbol length is less than the channel length. Therefore, in [18], the authors only analyzed the case $N \geq(D+1)$. Here, for V-OFDM, $N$ still needs to satisfy ${ }^{2} N \geq(D+1)$, however, $M$ can be a number less than, equal to or greater than the channel length $D+1$.

\section{B. Outage and ser Analysis for MMSE-V-OFDM}

The following lemma proved in [18] is useful for the diversity order analysis. For completeness, we state it here again without proof.

${ }^{2}$ Compared with both OFDM and SC-FDE, one of the advantages of V-OFDM is that we can use large symbol length $N$, and adjust $M$ for different requirements, thus the $\mathrm{CP}$ overhead can be reduced. 
Lemma 2: Assuming that the elements in $\mathbf{h}=$ $\left[h_{0}, h_{1}, \ldots, h_{D}\right]^{T}$ are i.i.d. zero mean complex Gaussian distributed, and a real constant $k \in(0, D+1)$, we have

$$
P\left[\sum_{d=0}^{D} \frac{1}{1+\rho\left|h_{d}\right|^{2}}>k\right] \doteq \rho^{-(\lfloor k\rfloor+1)}
$$

As stated before, in [18], the authors only considered the case $N \geq(D+1)$. In the following, we firstly consider the case $M \geq(D+1)$, which is similar as the case $N \geq(D+1)$ in [18]. Here, we just state the results for V-OFDM. In the proof, we briefly transform the problem to be the same as that in [18], and then apply the results in [18].

Lemma 3: Assume that the channel CIR h $=\left[h_{0}, h_{1}, \ldots, h_{D}\right]^{T}$ has i.i.d. zero mean complex Gaussian distributed elements. Define $\overline{\mathbf{h}}_{l} \triangleq\left[H_{l}, H_{l+L}, \ldots, H_{l+(M-1) L}\right]^{T}=\mathbf{Q}_{l} \mathbf{h}$, where $M \geq(D+1)$ and $\mathbf{Q}_{l}$ is an $M \times(D+1)$ matrix, whose $m$ th row and $d$ th column is defined as $\left[\mathbf{Q}_{l}\right]_{m, d}=\frac{1}{\sqrt{M}} \exp \left(-j 2 \pi(l+m L) \frac{d}{N}\right)$, where $N=M L$, $L \in \mathcal{N}$, and $\mathcal{N}$ means the set of natural numbers. The indices $m, d$, and $l$ have the range $m=0,1, \ldots, M-1$, $d=0,1, \ldots, D$, and $l=0,1, \ldots, L-1$, respectively. Then, for a real constant $k \in(0, D+1)$, we have

$$
P\left[\sum_{d=0}^{D} \frac{1}{1+\rho\left|h_{d}\right|^{2}}>k\right] \doteq P\left[\sum_{n=0}^{M-1} \frac{1}{1+\rho\left|H_{l+n L}\right|^{2}}>k\right]
$$

where $l=0,1, \ldots, L-1$.

Proof: Based on Lemma 1 we have that the PDF of $\overline{\mathbf{h}}_{l}$ does not depend on $l$, so in the following, without loss of generality, we assume that $l=0$, i.e., we only consider $\overline{\mathbf{h}}_{0}$ with $\left[\overline{\mathbf{h}}_{0}\right]_{m}=$ $H_{m L}$ and $\mathbf{Q}_{0}$ with $\left[\mathbf{Q}_{0}\right]_{m, d}=\frac{1}{\sqrt{M}} \exp \left(\frac{-j 2 \pi m d}{M}\right)$.

Define the size $(D+1)$ DFT of $\mathbf{h}$ as $\tilde{\mathbf{h}} \triangleq\left[\tilde{H}_{0}, \tilde{H}_{1}, \ldots, \tilde{H}_{D}\right]^{T}$. The elements in $\mathbf{h}$ are i.i.d. Gaussian distributed, so are the elements in $\tilde{\mathbf{h}}$. Thus we have

$$
P\left[\sum_{d=0}^{D} \frac{1}{1+\rho\left|h_{d}\right|^{2}}>k\right] \doteq P\left[\sum_{d=0}^{D} \frac{1}{1+\rho\left|\tilde{H}_{d}\right|^{2}}>k\right]
$$

for $k \in(0, D+1)$. So, we just need to prove that

$$
P\left[\sum_{d=0}^{D} \frac{1}{1+\rho\left|\tilde{H}_{d}\right|^{2}}>k\right] \doteq P\left[\sum_{n=0}^{M-1} \frac{1}{1+\rho\left|H_{l+n L}\right|^{2}}>k\right]
$$

Following the same approach as in [18], we firstly assume that $M=(D+1) K$, where $K \in \mathcal{N}$. In this case, $\overline{\mathbf{h}}_{0}=\mathbf{Q}_{0} \mathbf{h}$ is just the size- $M$ DFT of $\mathbf{h}$. When $m=d K(d=0,1, \ldots, D)$, we have $H_{m L}=H_{d K L}=\tilde{H}_{d}$. When $m \neq d K, H_{m L}$ can be interpolated from $\left\{\tilde{H}_{d}\right\}_{d=0}^{D}$ i.e.

$$
H_{m L}=\sum_{d=0}^{D} \tilde{H}_{d} \cdot \frac{1-e^{-j \frac{2 \pi m(D+1)}{M}}}{1-e^{-j \frac{2 \pi(m-d)}{M}}} .
$$

Then, following the same procedure as proving the Lemma 2 in [18], we can prove that, for both $M=(D+1) K$ and $M \neq$ $(D+1) K$, and any value $k \in(0, D+1)$, we have (19).

Because $N$ is a multiple of $M$, the assumption $M \geq(D+1)$ implies that $N \geq(D+1)$. Based on Lemma 3, we have the following theorem which shows the outage diversity of MMSE-VOFDM when $M \geq(D+1)$.

Theorem 3: For MMSE-V-OFDM, if $M \geq(D+1)$, the outage diversity $d_{\text {out }}^{M M S E}(R, M, D, N)$ equals

$$
d_{\text {out }}^{M M S E}(R, M, D, N)= \begin{cases}D+1, & \text { if } R \leq \log \frac{M}{D} \\ \left\lfloor 2^{-R} M\right\rfloor+1, & \text { if } R>\log \frac{M}{D}\end{cases}
$$

Proof: This can be proved the same way as that in Theorem 2 and Corollary 1 in [18].

Since in V-OFDM, the VB size $M$ can be a number smaller than the channel length, in the following we consider the case $M<(D+1)$. It is not a straightforward extension of the results in [18]. The properties of the matrix $\mathbf{Q}_{l}$ needs to be considered carefully.

Lemma 4: Assume that the channel CIR h $=\left[h_{0}, h_{1}, \ldots, h_{D}\right]^{T}$ has i.i.d. zero mean complex Gaussian distributed elements. Define $\overline{\mathbf{h}}_{l} \triangleq\left[H_{l}, H_{l+L}, \ldots, H_{l+(M-1) L}\right]^{T}=\mathbf{Q}_{l} \mathbf{h}$, where $\mathbf{Q}_{l}$ is defined the same as that in Lemma 3, except that $M<(D+1)$. Assume a real constant $k \in(0, M)$, then we have

$$
P\left[\sum_{n=0}^{M-1} \frac{1}{1+\rho\left|H_{l+n L}\right|^{2}}>k\right] \doteq \rho^{-(\lfloor k\rfloor+1)} .
$$

Proof: Similar to Lemma 3, based on Lemma 1, we only consider $l=0$.

If $(D+1)=K M$ and $K \in \mathcal{N}$, then $(D+1)$ can be divided by $M$ and $\mathbf{Q}_{0} \mathbf{Q}_{0}^{H}=K \mathbf{I}$. After the linear transformation, $\overline{\mathbf{h}}_{0}$ has $M$ i.i.d. zero mean Gaussian distributed elements. Based on Lemma 2, we have that if $k \in(0, M),(22)$ is proven.

If $(D+1)=K M+S$, where $K \in \mathcal{N}, S \in \mathcal{N}$, and $S \leq(M-1)$, then the elements in $\overline{\mathbf{h}}_{0}$ are correlated and the correlation matrix is

$$
\mathbf{R}_{\overline{\mathbf{h}}_{0}}=\mathbb{E}\left[\overline{\mathbf{h}}_{0} \overline{\mathbf{h}}_{0}^{H}\right]=\mathbf{Q}_{0} \mathbb{E}\left[\mathbf{h h}^{H}\right] \mathbf{Q}_{0}^{H}=\mathbf{Q}_{0} \mathbf{Q}_{0}^{H} .
$$

The singular value decomposition of $\mathbf{R}_{\overline{\mathbf{h}}_{0}}$ is

$$
\mathbf{R}_{\overline{\mathbf{h}}_{0}}=\mathbf{U} \Sigma \mathbf{U}^{H}
$$

where $\mathbf{U}$ is a unitary matrix, $\Sigma=\operatorname{diag}\left\{\sigma_{0}^{2}, \sigma_{1}^{2}, \ldots, \sigma_{M-1}^{2}\right\}$, and $\sigma_{0} \geq \sigma_{1} \geq \cdots \geq \sigma_{M-1}$. Because of the special structure of $\mathbf{Q}_{0}$, it is not difficult to verify that $\sigma_{0}=\sigma_{1}=\cdots=\sigma_{S-1}$ and $\sigma_{S}=\sigma_{S+1}=\cdots=\sigma_{M-1}$, and $\frac{\min \left\{\sigma_{n}^{2}\right\}}{\max \left\{\sigma_{n}^{2}\right\}}=\frac{\sigma_{S}^{2}}{\sigma_{0}^{2}}=\frac{K}{K+1}$.

Define $\hat{\mathbf{h}}_{0} \triangleq\left[\hat{H}_{0}, \hat{H}_{1}, \ldots, \hat{H}_{M-1}\right]^{T}=\mathbf{U}^{H} \overline{\mathbf{h}}_{0}$. After the unitary transformation, the elements in $\hat{\mathbf{h}}_{0}$ are independent. Because of the special structure of $\Sigma$, we have 
$\operatorname{var}\left(\hat{H}_{0}\right)=\operatorname{var}\left(\hat{H}_{1}\right)=\cdots=\operatorname{var}\left(\hat{H}_{S-1}\right)=\sigma_{0}^{2}$ and $\operatorname{var}\left(\hat{H}_{S}\right)=\operatorname{var}\left(\hat{H}_{1}\right)=\cdots=\operatorname{var}\left(\hat{H}_{M-1}\right)=\frac{K}{K+1} \sigma_{0}^{2}$.

Define two sequences $\left\{\hat{H}_{n}^{\prime}\right\}_{n=0}^{M-1}$ and $\left\{\hat{H}_{n}^{\prime \prime}\right\}_{n=0}^{M-1}$ as

$$
\hat{H}_{n}^{\prime}= \begin{cases}\hat{H}_{n}, & 0 \leq n \leq S-1 \\ \sqrt{\frac{K+1}{K}} \hat{H}_{n}, & S \leq n \leq M-1,\end{cases}
$$

and

$$
\hat{H}_{n}^{\prime \prime}= \begin{cases}\sqrt{\frac{K}{K+1}} \hat{H}_{n}, & 0 \leq n \leq S-1 \\ \hat{H}_{n}, & S \leq n \leq M-1,\end{cases}
$$

respectively. We can see that the elements in $\left\{\hat{H}_{n}^{\prime}\right\}_{n=0}^{M-1}$ and $\left\{\hat{H}_{n}^{\prime \prime}\right\}_{n=0}^{M-1}$ are i.i.d. Gaussian distributed. Based on Lemma 2, we have

$$
\begin{aligned}
\rho^{-(\lfloor k\rfloor+1)} & \doteq P\left[\sum_{n=0}^{M-1} \frac{1}{1+\rho\left|\hat{H}_{n}^{\prime \prime}\right|^{2}}>k\right] \\
& \leq P\left[\sum_{n=0}^{M-1} \frac{1}{1+\rho\left|\hat{H}_{n}\right|^{2}}>k\right] \\
& \leq P\left[\sum_{n=0}^{M-1} \frac{1}{1+\rho\left|\hat{H}_{n}^{\prime}\right|^{2}}>k\right] \doteq \rho^{-(\lfloor k\rfloor+1)}
\end{aligned}
$$

which means that

$$
P\left[\sum_{n=0}^{M-1} \frac{1}{1+\rho\left|\hat{H}_{n}\right|^{2}}>k\right] \doteq \rho^{-(\lfloor k\rfloor+1)},
$$

for $k \in(0, M)$.

So next we prove that

$$
P\left[\sum_{n=0}^{M-1} \frac{1}{1+\rho\left|\hat{H}_{n}\right|^{2}}>k\right] \doteq P\left[\sum_{n=0}^{M-1} \frac{1}{1+\rho\left|H_{n L}\right|^{2}}>k\right] .
$$

First, define

$$
\hat{\alpha}_{k}=-\frac{\log \left|\hat{H}_{k}\right|^{2}}{\log \rho} \text { and } \alpha_{k}=-\frac{\log \left|H_{k L}\right|^{2}}{\log \rho} .
$$

Because $\hat{\mathbf{h}}_{0}=\mathbf{U}^{H} \overline{\mathbf{h}}_{0}$, defining $u_{i j}=\left[\mathbf{U}^{H}\right]_{i j}$, then $\hat{H}_{k}=$ $\sum_{n=0}^{M-1} u_{k n} H_{n L}$, and

$$
\left|\hat{H}_{k}\right|^{2}=\sum_{n=0}^{M-1}\left|u_{k n}\right|^{2}\left|H_{n L}\right|^{2}+\underbrace{\sum_{i=0}^{M-1} \sum_{j=0, j \neq i}^{M-1} u_{k i} u_{k j}^{*} H_{i L} H_{j L}^{*}}_{\triangleq A},
$$

for $k=0,1, \ldots, M-1$. Because $u_{i j}$ is a constant, when $u_{i j} \neq$ 0 we have

$$
\begin{aligned}
\lim _{\rho \rightarrow \infty} \frac{\log \left|u_{k i}\right|^{2}\left|H_{i L}\right|^{2}}{\log \rho} & =\lim _{\rho \rightarrow \infty} \frac{\log \left|u_{k i}\right|^{2}+\log \left|H_{i L}\right|^{2}}{\log \rho} \\
& =\lim _{\rho \rightarrow \infty} \frac{\log \left|H_{i L}\right|^{2}}{\log \rho},
\end{aligned}
$$

i.e., $\left|u_{k n}\right|^{2}\left|H_{n L}\right|^{2} \doteq\left|H_{n L}\right|^{2} \doteq \rho^{-\alpha_{n}}$. Regarding the second item in the right-hand-side of (24), we define $\alpha_{A}=-\frac{\log |A|}{\log \rho}$. Then, (24) can be written as

$$
\begin{aligned}
\left|\hat{H}_{k}\right|^{2} & =\rho^{-\hat{\alpha}_{k}} \doteq \sum_{n=0}^{M-1} \rho^{-\alpha_{n}}+\frac{A}{|A|} \rho^{-\alpha_{A}} \\
& \doteq \rho^{-\min _{n} \alpha_{n}}+\frac{A}{|A|} \rho^{-\alpha_{A}} .
\end{aligned}
$$

First of all, the $A$ defined in (24) is real. Because the left-handside of (25) is positive, if $A<0$, we should have $\alpha_{A} \geq$ $\min _{n} \alpha_{n}$, which means that the second item of the right-hand side of (25) diminishes as $\rho \rightarrow \infty$ compared with the first item. So, when $A<0, \rho^{-\hat{\alpha}_{k}} \doteq \rho^{-\min _{n} \alpha_{n}}$. When $A>0, \rho^{-\hat{\alpha}_{k}} \doteq$ $\rho^{-\min _{n} \alpha_{n}}+\rho^{-\alpha_{A}}>\rho^{-\min _{n} \alpha_{n}}$. So we have

$$
\hat{\alpha}_{k} \leq \min _{n} \alpha_{n} .
$$

Because $\mathbf{U}$ is unitary, we have $\overline{\mathbf{h}}_{0}=\mathbf{U} \hat{\mathbf{h}}_{0}$. Following the same procedure as above, we have

$$
\alpha_{k} \leq \min _{n} \hat{\alpha}_{n}
$$

Then we have

$$
\begin{aligned}
P & {\left[\sum_{n=0}^{M-1} \frac{1}{1+\rho\left|\hat{H}_{n}\right|^{2}}>k\right] } \\
& =P\left[\sum_{n=0}^{M-1} \frac{1}{1+\rho^{1-\hat{\alpha}_{n}}}>k\right] \\
& \leq P\left[\sum_{n=0}^{M-1} \frac{1}{1+\rho^{1-\min _{i} \alpha_{i}}}>k\right] \\
& \leq P\left[\sum_{n=0}^{M-1} \frac{1}{1+\rho^{1-\alpha_{i}}}>k\right] \\
& \leq P\left[\sum_{n=0}^{M-1} \frac{1}{1+\rho\left|H_{n L}\right|^{2}}>k\right],
\end{aligned}
$$

for $k \in(0, M)$. Similarly,

$$
P\left[\sum_{n=0}^{M-1} \frac{1}{1+\rho\left|H_{n L}\right|^{2}}>k\right] \leq P\left[\sum_{n=0}^{M-1} \frac{1}{1+\rho\left|\hat{H}_{n}\right|^{2}}>k\right],
$$

for $k \in(0, M)$. Thus, (23) and the Lemma is proven.

Based on Lemma 4, we have the following theorem.

Theorem 4: For MMSE-V-OFDM, if $M \leq(D+1)$, the outage diversity order equals

$$
d_{\text {out }}^{M M S E}(R, M, D, N)=\left\lfloor M 2^{-R}\right\rfloor+1,
$$

regardless of the value of $N$.

Proof: For MMSE-V-OFDM, using $P_{\text {out }}(R, M, D, N)$ is

$$
\begin{aligned}
& P_{\text {out }}(R, M, D, N) \\
& \quad=P\left[I^{M M S E}\left(\hat{\mathbf{y}}, \mathbf{x}_{l}\right)<R\right] \\
& \quad=P\left[-\log \left(\frac{1}{M} \sum_{n=0}^{M-1} \frac{1}{1+\rho\left|H_{l+n L}\right|^{2}}\right)<R\right] \\
& \quad=P\left[\sum_{n=0}^{M-1} \frac{1}{1+\rho\left|H_{l+n L}\right|^{2}}>M 2^{-R}\right] .
\end{aligned}
$$


Since $R>0, M 2^{-R}<M$, using Lemma 4, we have $P_{\text {out }}(R, M, D, N) \doteq \rho^{-\left(\left\lfloor M 2^{-R}\right\rfloor+1\right)}$, and

$$
d_{\text {out }}^{M M S E}(R, M, D, N)=\left\lfloor M 2^{-R}\right\rfloor+1 .
$$

We can see that the diversity order is independent of $N$.

Combining Theorem 3 and Theorem 4, we have the following corollary.

Corollary 1: For MMSE-V-OFDM, the outage diversity $d_{\text {out }}^{M M S E}(R, M, D, N)$ equals

$$
d_{\text {out }}^{M M S E}(R, M, D, N)=\min \left\{\left\lfloor M 2^{-R}\right\rfloor, D\right\}+1,
$$

regardless of the V-OFDM symbol length $N$.

Proof: If $M \geq(D+1)$, Theorem 3 can be represented in a compact way as $d_{o u t}^{M M S E}(R, M, D, N)=$ $\min \left\{\left\lfloor M 2^{-R}\right\rfloor, D\right\}+1$.

If $M<(D+1)$, because $R>0,\left\lfloor M 2^{-R}\right\rfloor \leq D$. So, $\min \left\{\left\lfloor M 2^{-R}\right\rfloor, D\right\}+1=\left\lfloor M 2^{-R}\right\rfloor+1$, which means (26) is also valid for $M<(D+1)$.

The above results are for the outage diversity order. For the diversity order based on the SER, we have the following theorem. It is similar to that established in [18].

Theorem 5: For MMSE-V-OFDM, the diversity $d^{M M S E}(R, M, D, N)$ equals

$$
d^{M M S E}(R, M, D, N)=\min \left\{\left\lfloor M 2^{-R}\right\rfloor, D\right\}+1 \text {. }
$$

Proof: Using arguments similar to those in Lemma 3 in [18], we can prove the upper bound, i.e., $d_{\text {out }}^{M M S E}(R, M, D, N) \geq d^{M M S E}(R, M, D, N)$. Also using similar arguments as those in Lemma 4 in [18], and noticing the special structure of $\mathbf{Q}_{0}$, we can prove the lower bound $d_{\text {out }}^{M M E}(R, M, D, N) \leq d^{M M S E}(R, M, D, N)$. Combining both the upper bound and lower bound, we can prove the theorem.

Compared to the results of the diversity obtained in [18] for SC-FDE, the above results are their generalizations. For SC-FDE, the transmission block length $L$ in [18] has to be more than the channel length $D+1$, while the VB size $M$ in this paper can be smaller than $D+1$.

\section{Outage and Ser Analysis For ZF-V-OFDM}

If $\mathrm{ZF}$ receiver is used for $\mathrm{V}-\mathrm{OFDM}$, the diversity order only equals 1 . This is summarized in the following theorem.

Theorem 6: For ZF-V-OFDM, the diversity order $d^{Z F}(R, M, D, N)=1$.

Proof: Substituting (14) into $P_{\text {out }}(R, M, D, N)(18)$ and following the same procedure as that used in [18] to prove the Theorem 4, we can show that $d_{\text {out }}^{Z F}(R, M, D, N)=1$. Similarly, we can show that $d^{Z F}(R, M, D, N)=1$.

\section{Simulation Results}

In this section, we do simulations to validate the analysis. In all the simulations, we assume that the V-OFDM symbol length $N=1024$, the CP length $P=128$, and the channel CIR is a length $D+1$ vector which has i.i.d. complex Gaussian distributed elements. For all the SER plots, the system is uncoded.

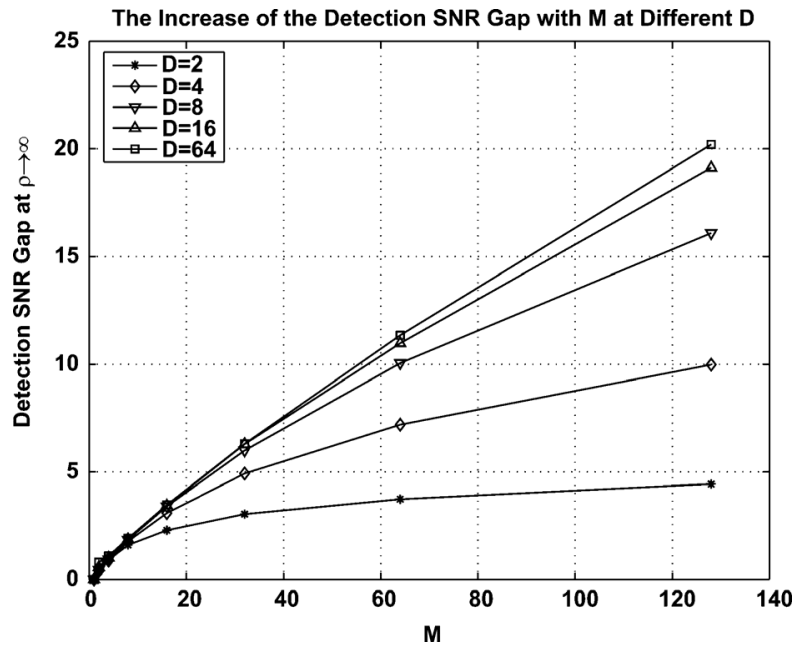

Fig. 2. Increase of the detection SNR gap at $\rho \rightarrow \infty$ with $M$ at different $D$.

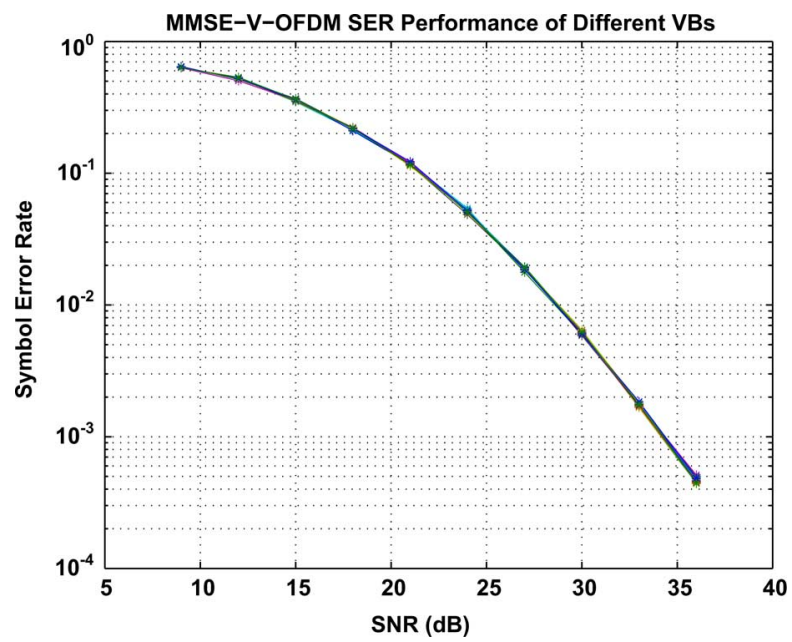

Fig. 3. MMSE-V-OFDM SER performance of different VBs at $M=64, D=$ 2 and 16QAM.

$R=2$, 4, and 6 represent QPSK, 16QAM, and 64QAM, respectively.

A. The Increase of the Detection SNR Gap $\mathbb{E}_{\mathbf{H}_{l}}\left[\rho_{l}^{m m s e}-\rho_{l}^{z f}\right]$ With the VB Size $m$

In Fig. 2, the value $\mathbb{E}_{\mathbf{H}_{l}}\left[\rho_{l}^{M M S E}-\rho_{l}^{Z F}\right]$ is plotted versus $M$ at different channel lengths $D$. From the plot, we can clearly see that the gain of the MMSE-V-OFDM over the ZF-V-OFDM increases monotonically with the VB size $M$. Also, as $D$ increases, the gain increases as well. Please note that this detection SNR gap cannot be simply mapped to the SNR gap in the SER versus SNR curves below, where in the SER versus SNR curves the gap is between the channel SNRs.

\section{B. The Equal Performance of Different VBs}

To verify Theorem 2, Fig. 3 plots the SER versus SNR for different VBs when MMSE-V-OFDM is used. The parameters assumed in the plots are: $(R, M)=(4,64)$, and $D=2$. In this case, there are totally $L=\frac{N}{M}=\frac{1024}{64}=16 \mathrm{VBs}$. In the figure, we plot 16 curves. The equal performance can be clearly seen from the overlaps of the curves for different VBs. Also, it 


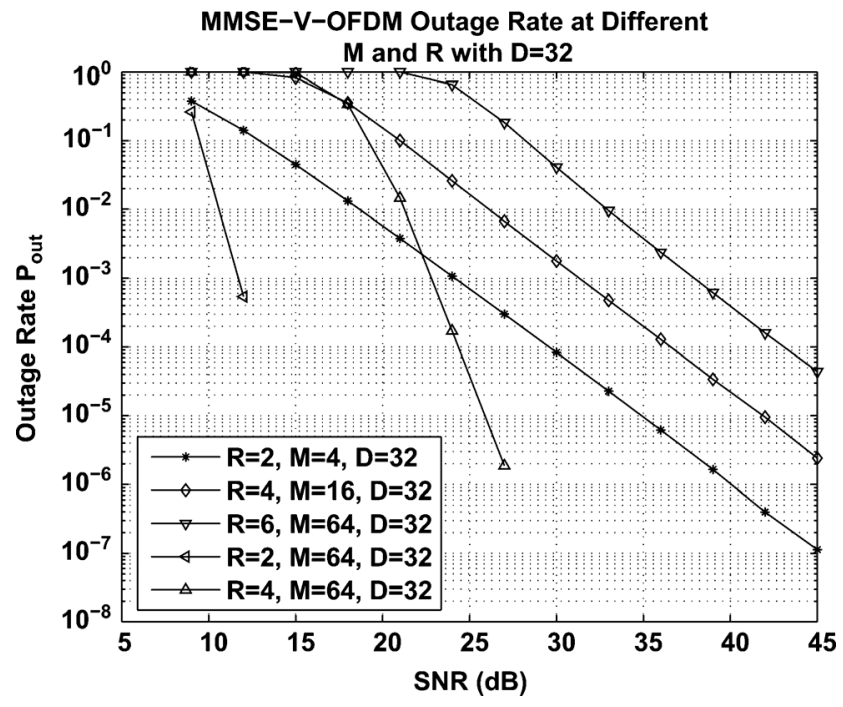

Fig. 4. MMSE-V-OFDM outage rate at different $R$ and $M$ with $D=32$.

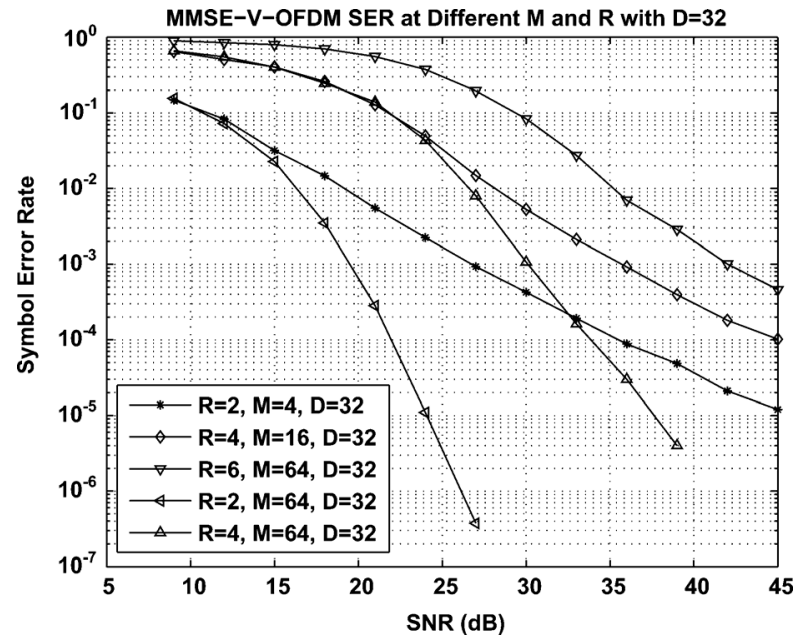

Fig. 5. MMSE-V-OFDM SER at different $R$ and $M$ with $D=32$.

is worth noticing that for ML-V-OFDM, due to the complexity, it is difficult to run simulation for large $M$, while for MMSE-VOFDM, we can get the simulation results for large $M$ easily. For ZF-V-OFDM, we have similar results.

\section{The MMSE-V-OFDM Diversity Order}

Figs. 4 and 5 show the MMSE-V-OFDM outage rate $P_{\text {out }}$ and SER at $D=32$ for different $M$ and $R$. For all the cases in Figs. 4 and 5, $\left\lfloor M 2^{-R}\right\rfloor<D$. According to Corollary 1 and Theorem 5, the diversity order equals $d_{\text {out }}^{M M S E}=d^{M M S E}=$ $\left\lfloor M 2^{-R}\right\rfloor+1$. When $(R, M)=(2,4),(R, M)=(4,16)$, and $(R, M)=(6,64)$, the diversity orders are the same, i.e., $d_{\text {out }}^{M M S E}=d^{M M S E}=2$. This can be clearly seen from the slopes of the curves for these three cases from Figs. 4 and 5. Comparing the cases $(R, M)=(2,4)$ and $(R, M)=(2,64)$ and the cases $(R, M)=(4,16)$ and $(R, M)=(4,64)$ in both Figs. 4 and 5, we can see the increase of the diversity order with $M$. Also, comparing the cases $(R, M)=(2,64)$, $(R, M)=(4,64)$, and $(R, M)=(6,64)$, we can see the decrease of the diversity order with $R$.

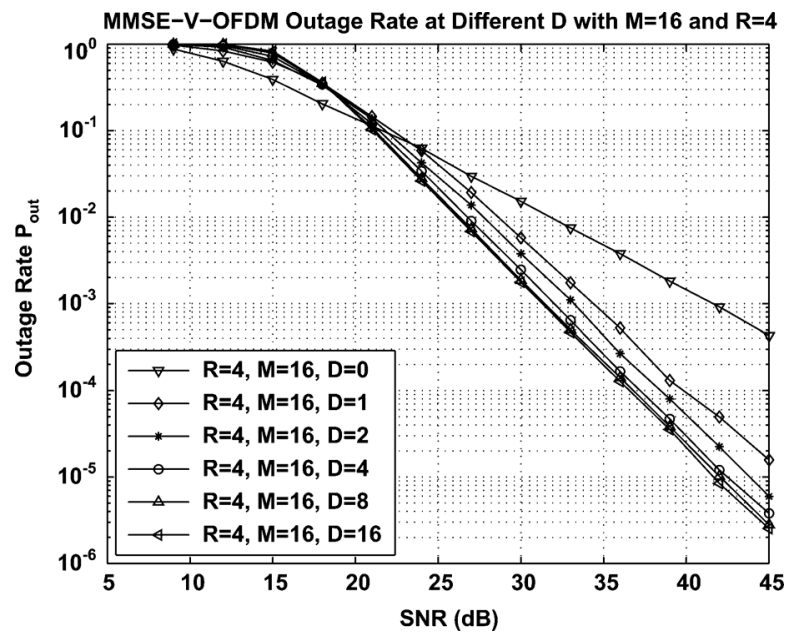

Fig. 6. MMSE-V-OFDM outage rate at different $D$ with $M=16$ and $R=4$.

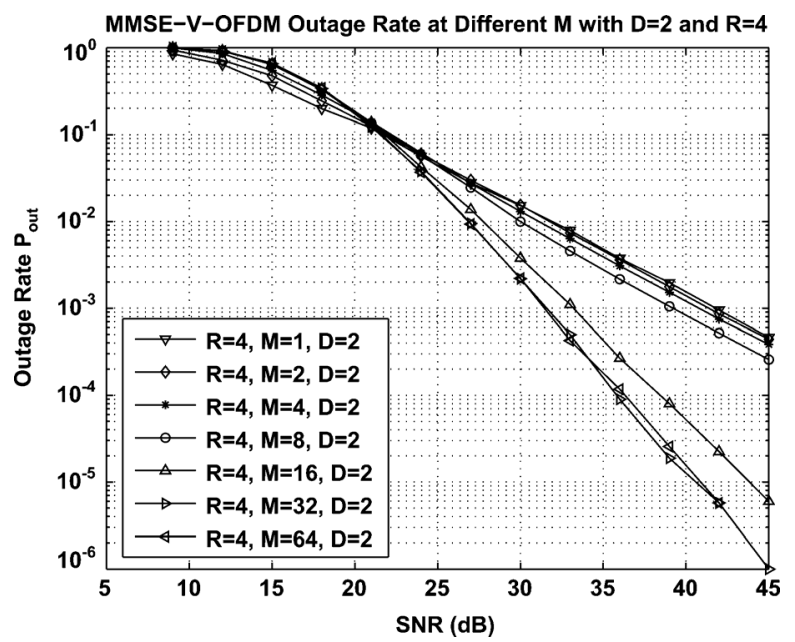

Fig. 7. MMSE-V-OFDM outage rate at different $M$ with $D=2$ and $R=4$.

Fig. 6 compares the outage rate at different CIR length $D+1$ with $(R, M)=(4,16)$, in this case $\left\lfloor M 2^{-R}\right\rfloor=1$. From the plot we can see that, when $D=0$, the diversity order is limited by the channel, and is only 1 . When $D$ is increased from 0 to 1 , the slope of the curve changes. When $D$ is greater than 1 , the diversity order is limited by $R$ and $M$, and we can only see a small shift of the curve and the slope does not change. This matches with the theoretical analysis.

Fig. 7 compares the outage rate at different $M$ with $R=4$ and $D=2$. When $M=1,2,4,8,\left\lfloor M 2^{-R}\right\rfloor=0$ and the diversity order is limited by $M$ and $R$. And the curves show the same slope for these cases. When $M=16$ and 32, $\left\lfloor M 2^{-R}\right\rfloor=$ 1 and 2, respectively. From the curves we can see the increase of the slope for these two cases. When $M=64,\left\lfloor M 2^{-R}\right\rfloor=4$ and the diversity order is limited by $D$. So, the diversity order still equals 2. From the curve we can see the same slope of the cases $M=32$ and $M=64$. This verifies the theoretical analysis.

Fig. 8 compares the SER of ML-V-OFDM and MMSE-VOFDM at different $M$ with $R=2$ and $D=32$. For ML-VOFDM, when $M$ increases from 1 to 4 , the diversity order of the majority of the VBs increases [12], [16] [17], and the performance increase can be seen from Fig. 8. For MMSE-V-OFDM, 


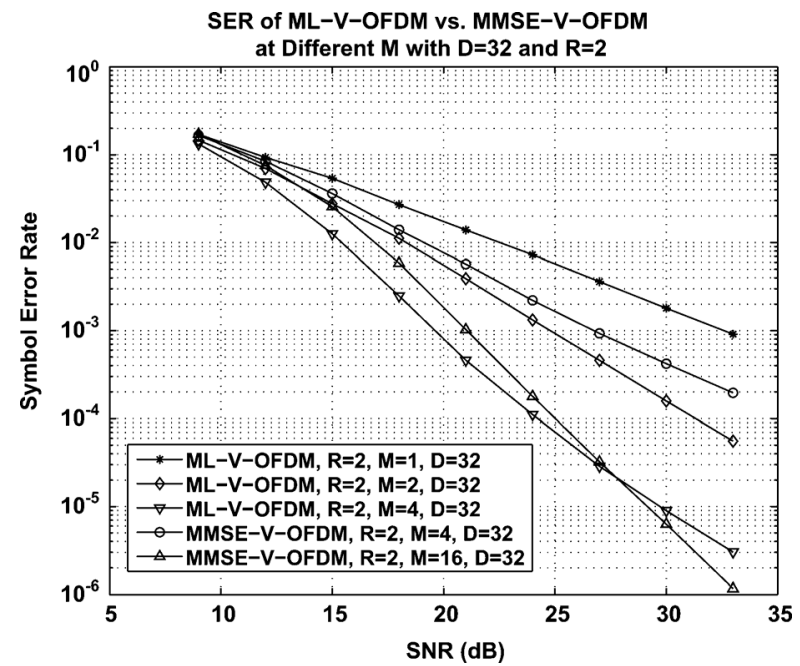

Fig. 8. SER of ML-V-OFDM versus MMSE-V-OFDM at different $M$ with $D=32$ and $R=2$.

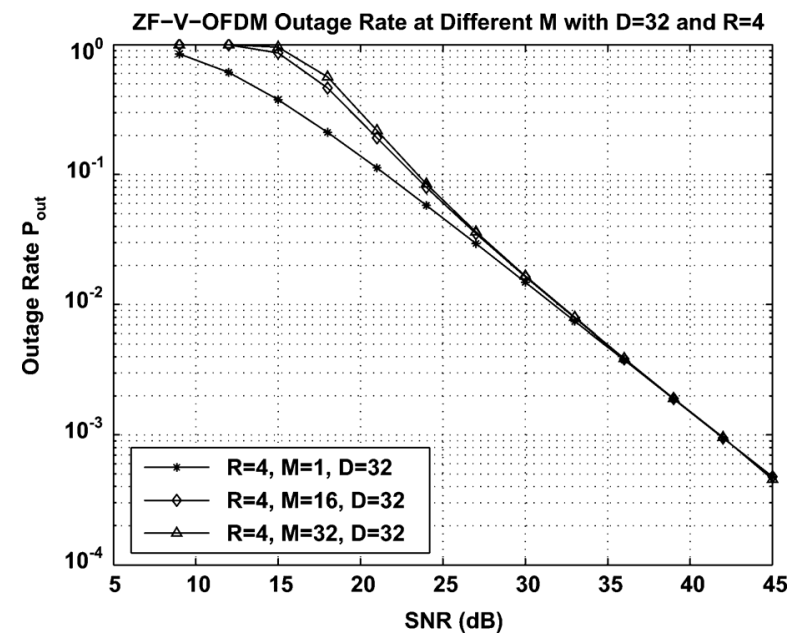

Fig. 9. ZF-V-OFDM outage rate at different $M$.

we simulate the cases $M=4$ and $M=16$. When MMSE-VOFDM is at $M=4$, the diversity order equals 2 . From Fig. 8 , we can see that its performance is more close to the ML-VOFDM at $M=2$, whose majority of the VBs have diversity order of 2 . When MMSE-V-OFDM is at $M=16$, the diversity order equals 5. From Fig. 8, we can see that when SNR is high, MMSE-V-OFDM with $M=16$ outperforms ML-V-OFDM with $M=4$ whose majority of the VBs have diversity order of 4 . These confirm the theoretical analysis in the paper.

\section{The ZF-V-OFDM Diversity Order}

Fig. 9 shows the outage rate of ZF-V-OFDM at different $M$ with $D=32$ and $R=4$. We can see that ZF-V-OFDM does not provide any diversity gain.

Fig. 10 compares the SER performance of conventional OFDM (i.e., V-OFDM with $M=1$ ) MMSE-V-OFDM and ZF-V-OFDM at different $M$, with $D=32$ and $R=4$. From the plot, we can see that, when $M$ is increasing, the ZF-V-OFDM does not have any diversity gain, and it is equivalent to the conventional OFDM at high SNR. In comparison,

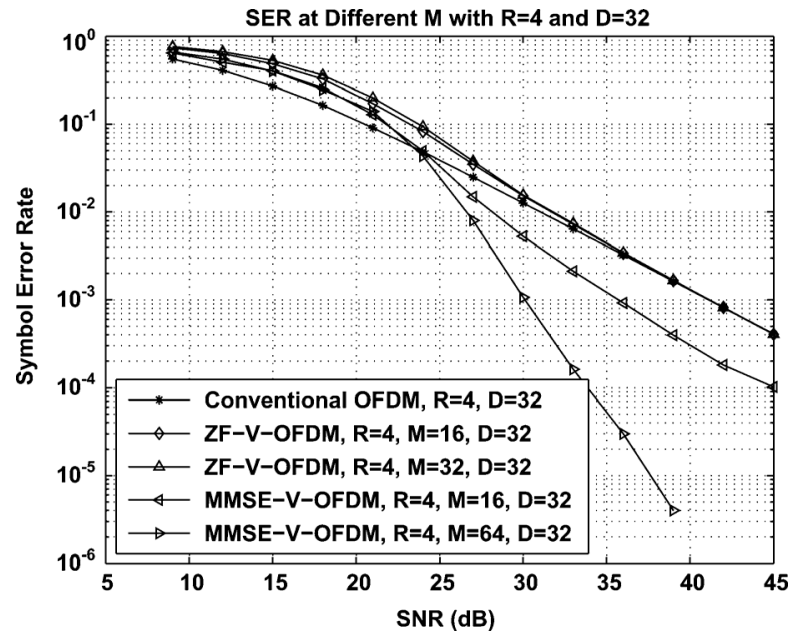

Fig. 10. SER comparison between conventional OFDM, MMSE-V-OFDM and ZF-V-OFDM.

for MMSE-V-OFDM, when $M=16$ and $64,\left\lfloor M 2^{-R}\right\rfloor=1$ and 4 , and the diversity order equals 2 and 5 , respectively. From the plot, we can clearly see the better performance of MMSE-V-OFDM.

\section{CONCLUSIONS}

In this paper, we analyzed the performance of V-OFDM with MMSE and ZF receivers. We showed that for both ZF-V-OFDM and MMSE-V-OFDM, all the VBs have the same performance. This is different from ML-V-OFDM. Also, different from ML-V-OFDM, the complexity of ZF-V-OFDM and MMSE-V-OFDM does not increase with the size of VB. With simply more information about the noise variance, MMSE-V-OFDM has better performance than ZF-V-OFDM and can provide diversity gain when the VB size increases. We analyzed the diversity orders for MMSE-V-OFDM. Also, we showed that ZF-V-OFDM does not provide any diversity gain. Due to the controllable PAPR and controllable performance, $\mathrm{V}-\mathrm{OFDM}$ is a promising practical transmission scheme. From the analysis of the paper, we can see that MMSE-V-OFDM could be more promising than ML-V-OFDM and ZF-V-OFDM in terms of the complexity and performance tradeoff.

APPENDIX A

DERIVATION OF (11)

$$
\begin{aligned}
\mathbb{E}\left[\hat{y}_{l M+m}^{M M S E}\left(\hat{y}_{l M+m}^{M M S E}\right)^{*}\right] & =\left[\mathbb{E}\left[\hat{\mathbf{y}}_{l}^{M M S E}\left(\hat{\mathbf{y}}_{l}^{M M S E}\right)^{H}\right]\right]_{m, m} \\
& =\frac{1}{M} \sum_{k=0}^{M-1} \frac{\left|H_{l+k L}\right|^{2}}{\left|H_{l+k L}\right|^{2}+\rho^{-1}} .
\end{aligned}
$$

We can see that it is independent of $m$.

$$
\begin{aligned}
\rho_{l, m}^{M M S E} & =\frac{\left|C_{l M+m}\right|^{2}}{\mathbb{E}\left[\hat{y}_{l M+m}^{M M S E}\left(\hat{y}_{l M+m}^{M M S E}\right)^{*}\right]-\left|C_{l M+m}\right|^{2}} \\
& =\left[\frac{1}{M} \sum_{k=0}^{M-1} \frac{1}{\rho\left|H_{l+k L}\right|^{2}+1}\right]^{-1}-1,
\end{aligned}
$$

for $m=0,1, \ldots, M-1$. 


\section{APPENDIX B \\ PROOF OF THEOREM 1}

Proof: Using (8), $\rho_{l}^{Z F}$ can be written as

$$
\rho_{l}^{Z F}=\frac{\rho}{\left[\left(\mathbf{H}_{l}^{H} \mathbf{H}_{l}\right)^{-1}\right]_{m, m}} .
$$

Since the diagonal elements of $\left(\mathbf{H}_{l}^{H} \mathbf{H}_{l}\right)^{-1}$, the right-hand-side of the above does not depend on $m$. Using $\mathbf{h}_{l, m}$ and $\mathbf{H}_{l, m}, \rho_{l, m}^{Z F}$ can also be written as

$$
\begin{aligned}
& \rho_{l}^{Z F}=\rho \\
& \quad \times\left[\mathbf{h}_{l, m}^{H} \mathbf{h}_{l, m}-\mathbf{h}_{l, m}^{H} \mathbf{H}_{l, m}\left(\mathbf{H}_{l, m}^{H} \mathbf{H}_{l, m}\right)^{-1} \mathbf{H}_{l, m}^{H} \mathbf{h}_{l, m}\right] .
\end{aligned}
$$

Similarly, Using (11), $\rho_{l}^{M M S E}$ can be written as

$$
\begin{aligned}
\rho_{l}^{M M S E} & =\rho \mathbf{h}_{l, m}^{H} \mathbf{h}_{l, m} \\
& -\rho \mathbf{h}_{l, m}^{H} \mathbf{H}_{l, m}\left(\mathbf{H}_{l, m}^{H} \mathbf{H}_{l, m}+\rho^{-1} \mathbf{I}\right)^{-1} \mathbf{H}_{l, m}^{H} \mathbf{h}_{l, m} .
\end{aligned}
$$

Then $\rho_{l, m}^{M M S E}-\rho_{l, m}^{Z F}$ is

$$
\begin{aligned}
\rho_{l}^{M M S E}-\rho_{l, m}^{Z F} & \\
= & \rho \mathbf{h}_{l, m}^{H} \mathbf{H}_{l, m}\left(\mathbf{H}_{l, m}^{H} \mathbf{H}_{l, m}\right)^{-1} \mathbf{H}_{l, m}^{H} \mathbf{h}_{l, m} \\
& \quad-\rho \mathbf{h}_{l, m}^{H} \mathbf{H}_{l, m}\left(\mathbf{H}_{l, m}^{H} \mathbf{H}_{l, m}+\rho^{-1} \mathbf{I}\right)^{-1}
\end{aligned}
$$

Do SVD of $\mathbf{H}_{l, m}$, i.e., $\mathbf{H}_{l, m}=\mathbf{U}_{l, m} \Lambda_{l, m} \mathbf{V}_{l, m}^{H}$, and substitute it into the above equation, we have

$$
\begin{aligned}
\rho_{l}^{M M S E}-\rho_{l}^{Z F} & \\
= & \rho \mathbf{h}_{l, m}^{H} \mathbf{U}_{l, m} \boldsymbol{\Lambda}_{l, m} \\
& \times\left[\boldsymbol{\Lambda}_{l, m}^{-2}-\left(\boldsymbol{\Lambda}_{l, m}^{2}+\rho^{-1} \mathbf{I}\right)^{-1}\right] \boldsymbol{\Lambda}_{l, m} \mathbf{U}_{l, m}^{H} \mathbf{h}_{l, m} \\
= & \mathbf{h}_{l, m}^{H} \mathbf{U}_{l, m}\left(\boldsymbol{\Lambda}_{l, m}^{2}+\rho^{-1} \mathbf{I}\right)^{-1} \mathbf{U}_{l, m}^{H} \mathbf{h}_{l, m} .
\end{aligned}
$$

So, as $\rho$ increases, $\rho_{l}^{M M S E}-\rho_{l}^{Z F}$ also increases, and

$$
\begin{aligned}
\lim _{\rho \rightarrow \infty}\left(\rho_{l}^{M M S E}-\rho_{l}^{Z F}\right) & =\mathbf{h}_{l, m}^{H} \mathbf{U}_{l, m} \boldsymbol{\Lambda}_{l, m}^{-2} \mathbf{U}_{l, m}^{H} \mathbf{h}_{l, m} \\
& =\left\|\mathbf{h}_{l, m}^{H} \mathbf{H}_{l, m}\left(\mathbf{H}_{l, m}^{H} \mathbf{H}_{l, m}\right)^{-1}\right\|^{2} .
\end{aligned}
$$

Using the representation of (27) and (28), it is not difficult to see that $\lim _{\rho \rightarrow \infty} \frac{\rho^{M M S E}}{\rho^{Z F}}=1$.

Since the left-hand side of the above equation is independent of $m$, the right-hand side is also independent of $m$.

\section{ACKNOWLEDGMENT}

The authors would like to thank the anonymous reviewers for their detailed and constructive comments that have improved the presentation of this paper.

\section{REFERENCES}

[1] L. J. Cimini, "Analysis and simulation of a digital mobile channel using orthogonal frequency division multiplexing," IEEE Trans. Commun, vol. COM-33, no. 7, pp. 665-675, Jul. 1985.

[2] J. A. C. Bingham, "Multicarrier modulation for data transmission: An idea whose time has come," IEEE Commun. Mag., vol. 28, no. 5, pp. 5-14, May 1990.
[3] $802.16 \mathrm{~m} / \mathrm{D} 5$, Draft Amendment to IEEE Standard for Local and Metropolitan Area Networks Part 16: Air Interface for Fixed and Mobile Broadband Wireless Access Systems.

[4] 3GPP TS 36.211, Evolved Universal Terrestrial Radio Access (E-UTRA), Physical Channels and Modulation, Release 10 10.1.0 2011-03.

[5] IEEE 802.11ad standard draft D0.1 [Online]. Available: http://www. ieee802.org/11/Reports/tgad update.htm

[6] S. H. Han and J. H. Lee, "An overview of peak-to-average power ratio reduction techniques for multicarrier transmission," IEEE Trans. Wireless Commun., vol. 12, no. 2, pp. 56-65, Apr. 2005.

[7] H. Sari, G. Karam, and I. Jeanclaude, "Transmission techniques for digital terrestrial TV broadcasting," IEEE Commun. Mag., pp. 100-109, Feb. 1995.

[8] M. V. Clark, "Adaptive frequency-domain equalization and diversity combining for broadband wireless communications," IEEE J. Sel. Areas Commun., vol. 16, pp. 1385-1395, Oct. 1998.

[9] N. Al-Dhahir, "Single-carrier frequency-domain equalization in frequency-selective fading channels," IEEE Commun. Lett., vol. 7, no. 7, pp. 304-306, Jul. 2001.

[10] D. Falconer, S. L. Ariyavisitakul, A. Benyamin-Seeyar, and B. Eidson, "Frequency domain equalization for single-carrier broadband wireless systems," IEEE Commun. Mag., vol. 40, no. 4, pp. 58-66, Apr. 2002.

[11] C. Ciochina and H. Sari, "A review of OFDMA and single-carrier FDMA," in Proc. 2010 Eur. Wireless Conf., Apr. 12-15, 2010, pp. 706-710.

[12] X.-G. Xia, "Precoded and vector OFDM robust to channel spectral nulls and with reduced cyclic prefix length in single transmit antenna systems," IEEE Trans. Commun., vol. 49, no. 8, pp. 1363-1374, Aug. 2001.

[13] H. Zhang, X.-G. Xia, L. J. Cimini, and P. C. Ching, "Synchronization techniques and guard-band-configuration scheme for single-antenna vector-OFDM systems," IEEE Trans. Wireless Commun., vol. 4, no. 5, pp. 2454-2464, Sep. 2005.

[14] H. Zhang and X.-G. Xia, "Iterative decoding and demodulation for single-antenna vector OFDM systems," IEEE Trans. Veh. Technol., vol. 55, no. 4, pp. 1447-1454, Jul. 2006.

[15] H. Zhang, X.-G. Xia, Q. Zhang, and W. Zhu, "Precoded OFDM with adaptive vector channel allocation for scalable video transmission over frequency-selective fading channels," IEEE Trans. Mobile Comput., vol. 1, no. 2, pp. 132-141, Apr.-Jun. 2002.

[16] C. Han, T. Hashimoto, and N. Suehiro, "Constellation-rotated vector OFDM and its performance analysis over Rayleigh fading channels," IEEE Trans. Commun., vol. 58, no. 3, pp. 828-837, Mar. 2010.

[17] P. Cheng, M. Tao, Y. Xiao, and W. Zhang, "V-OFDM: On performance limits over multi-path Rayleigh fading channels," IEEE Trans. Commun., vol. 59, no. 7, pp. 1878-1892, Jul. 2011.

[18] A. Tajer and A. Nosratinia, "Diversity order in ISI channels with singlecarrier frequency-domain equalizers," IEEE Trans. Wireless Commun., vol. 9, no. 3, pp. 1022-1032, Mar. 2010.

[19] S. H. Song and K. B. Lataief, "Diversity analysis for linear equalizers over ISI channels," IEEE Trans. Commun., vol. 59, no. 9, pp. 2414-2423, Sep. 2011.

[20] J. Liu, J.-K. Zhang, and K. M. Wong, "Full-diversity codes for MISO systems equipped with linear or ML detectors," IEEE Trans. Inf. Theory, vol. 54, no. 10, pp. 4511-4527, Oct. 2008.

[21] Y.-P. Lin and S.-M. Phoong, "BER minimized OFDM systems with channel independent precoders," IEEE Trans. Signal Process., vol. 51, no. 9, pp. 2369-2380, Sep. 2003.

[22] S. Ohno, "Performance of single-carrier block transmissions over multiplath fading channels with linear equalization," IEEE Trans. Signal Process., vol. 54, pp. 3678-3687, Oct. 2006.

[23] C. Tepedelenlioglu, "Maximum multipath diversity with linear equalization in precoded OFDM systems," IEEE Trans. Inf. Theory, vol. 50, no. 1, pp. 232-235, Jan. 2004.

[24] X. Ma and W. Zhang, "Fundamental limits of linear equalizers: Diversity, capacity, and complexity," IEEE Trans. Inf. Theory, vol. 54, no. 8, pp. 3442-3456, Aug. 2008.

[25] A. Hedayat and A. Nosratinia, "Outage and diversity of linear receivers in flat-fading MIMO channels," IEEE Trans. Signal Process., vol. 55, no. 12, pp. 5868-5873, Dec. 2007.

[26] N. Kim, Y. Lee, and H. Park, "Performance analysis of MIMO system with linear MMSE receiver," IEEE Trans. Wireless Commun., vol. 7, no. 11, pp. 4474-4478, Nov. 2008.

[27] K. R. Kumar, G. Caire, and A. L. Moustakas, "Asymptotic performance of linear receivers in MIMO fading channels," IEEE Trans. Inf. Theory, vol. 55 , no. 10 , pp. $4398-4418$, Oct. 2009. 
[28] Y. Jiang, M. K. Varanasi, and J. Li, "Performance analysis of ZF and MMSE equalizers for MIMO systems: An in-depth study of the high SNR regime," IEEE Trans. Inf. Theory, vol. 57, no. 4, pp. 2008-2026, Apr. 2011.

[29] A. H. Mehana and A. Nosratinia, "Diversity of MMSE MIMO receivers," in Proc. IEEE Int. Symp. Inf. Theory, Jun. 13-18, 2010, pp. 2163-2167.

[30] P. Li, D. Paul, R. Narasimhan, and J. Cioffi, "On the distribution of SINR for the MMSE MIMO receiver and performance analysis," IEEE Trans. Inf. Theory, vol. 52, no. 1, pp. 271-286, Jan. 2006.

[31] M. Vehkaperä and M. Juntti, "On the performance of space-time coded and spatially multiplexed MIMO systems with linear receivers," IEEE Trans. Commun., vol. 58, no. 2, pp. 642-651, Feb. 2010.

[32] A. L. Moustakas, "SINR distribution of MIMO MMSE receiver," in Proc. IEEE Int. Symp. Inf. Theory (ISIT), St. Petersburg, Russia, Jul.Aug. 31-5, 2011.

[33] X. Giraud, E. Boutillon, and J. C. Belfiore, "Algebraic tools to build modulation schemes for fading channels," IEEE Trans. Inf. Theory, vol. 43, no. 3, pp. 938-952, May 1997.

[34] J. Boutros and E. Viterbo, "Signal space diversity: A power- and bandwidth-efficient diversity technique for the Rayleigh fading channel," IEEE Trans. Inf. Theory, vol. 44, no. 4, pp. 1453-1467, Jul. 1998.

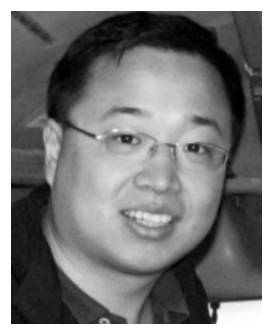

Yabo Li (M'05) received the B.S. and M.S. degrees in electrical engineering from Xi' an Jiaotong University, Xi'an, China, in 1998 and 2001, respectively, and the Ph.D. degree in electrical engineering from University of Delaware, Newark, in December 2005.

He was a member of Technical Staff with Bell-Labs (Beijing), Lucent Technologies (China), Beijing, from July 2001 to July 2002, and an intern with Mitisubishi Electric Research Labs, Cambridge, MA, from January 2005 to June 2005 . He was with the CDMA System Design Group of Nortel Networks, Richardson, TX, from December 2005 to May 2007, and with SiBEAM Inc., Sunnyvale, CA, from May 2007 to Dec. 2009. He is now with the Department of Information Science and Electronic Engineering of Zhejiang University, Hangzhou Zhejiang, China. His research interests include wireless communications, digital signal processing, and information theory.

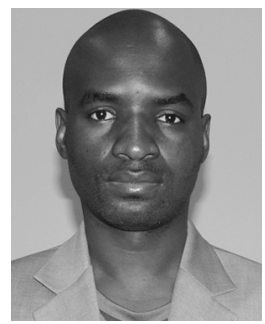

Ibo Ngebani received the B.S. degree in communications engineering from Queens University, Ontario, Canada, and the Masters degree in electrical and electronic engineering from the University of Botswana in 2003 and 2009 , respectively.

$\mathrm{He}$ is currently pursuing the $\mathrm{Ph} . \mathrm{D}$. degree at Zhejiang University, Hangzhou, China. From 2004 to 2010, he was with the Botswana Power Corporation working as a Telecommunications Engineer. His work included the provision of facilities for speech, teleprotection, and SCADA using power line carrier, fiber optic, and microwave radio communications. His research interests are in wireless communications theory with an emphasis on compensation for RF impairments and signal processing.

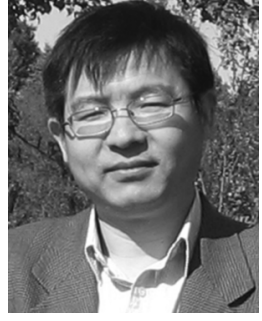

Xiang-Gen Xia (M'97-S'00-F'09) received the B.S degree in mathematics from Nanjing Normal University, Nanjing, China, and the M.S. degree in mathematics from Nankai University, Tianjin, China, and the $\mathrm{Ph} . \mathrm{D}$. degree in electrical engineering from the University of Southern California, Los Angeles, in 1983, 1986, and 1992, respectively.

He was a Senior/Research Staff Member at Hughes Research Laboratories, Malibu, CA, during 1995-1996. In September 1996, he joined the Department of Electrical and Computer Engineering, University of Delaware, Newark, where he is the Charles Black Evans Professor. His current research interests include space-time coding, MIMO and OFDM systems, digital signal processing, and SAR and ISAR imaging. $\mathrm{He}$ has more than 230 refereed journal articles published and accepted, and seven U.S. patents awarded. He is the author of the book Modulated Coding for Intersymbol Interference Channels (New York: Marcel Dekker, 2000).

Dr. Xia received the National Science Foundation (NSF) Faculty Early Career Development (CAREER) Program Award in 1997, the Office of Naval Research (ONR) Young Investigator Award in 1998, and the Outstanding Overseas Young Investigator Award from the National Nature Science Foundation of China in 2001. He also received the Outstanding Junior Faculty Award of the Engineering School of the University of Delaware in 2001. He is currently an Associate Editor of the IEEE TRANSACTIONS ON WIRELESS Communications, IEEE Transactions on Signal Processing, Signal Processing (China), and the Journal of Communications and Networks (JCN). He was a Guest Editor of Space-Time Coding and Its Applications in the EURASIP Journal of Applied Signal Processing in 2002. He served as an Associate Editor of the IEEE TRANSACTIONS ON Signal PROCESSING during 1996 to 2003, the IEEE TRANSACTIONS ON MOBILE COMPUTING during 2001 to 2004, IEEE TRANSACTIONS ON Vehicular TeChNOLOGY during 2005 to 2008, the IEEE Signal Processing LetTers during 2003 to 2007, Signal Processing (EURASIP) during 2008 to 2011, and the EURASIP Journal of Applied Signal Processing during 2001 to 2004. He served as a Member of the Signal Processing for Communications Committee from 2000 to 2005 and a Member of the Sensor Array and Multichannel (SAM) Technical Committee from 2004 to 2009 in the IEEE Signal Processing Society. He serves as IEEE Sensors Council Representative of IEEE Signal Processing Society (from 2002) and served as the Representative of IEEE Signal Processing Society to the Steering Committee for IEEE TRANSACTIONS ON MOBILE COMPUTING during 2005 to 2006. He is the Technical Program Chair of the Signal Processing Symposium, Globecom 2007 in Washington DC, and the General Co-Chair of ICASSP 2005 in Philadelphia.

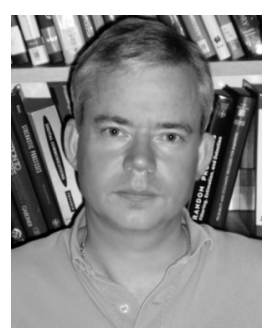

Anders Høst-Madsen (M'95-SM'02) was born in Denmark in 1966. He received the M.Sc. degree in engineering in 1990 and the Ph.D. degree in mathematics in 1993, both from the Technical University of Denmark.

From 1993 to 1996, he was with Dantec Measurement Technology A/S, Copenhagen, Denmark, from 1996 to 1998 he was an Assistant Professor at Kwangju Institute of Science and Technology, Kwangju, Korea, and from 1998 to 2000 an Assistant Professor with the Department of Electrical and Computer Engineering, University of Calgary, Calgary, AB, Canada, and a Staff Scientist at TRLabs, Calgary. Since 2001, he has been with the Department of Electrical Engineering, University of Hawaii at Manoa, Honolulu, and a Professor there since 2009. He was also a founder and CTO (2007-2008) of Kai Medical, Inc., which is making equipment for noncontact heart monitoring. His research interests are in statistical signal processing, information theory, and wireless communications, including ad-hoc networks, cooperative diversity, wireless sensor networks, heart monitoring, life detection, and marine mammal signal processing.

Dr. Høst-Madsen has served as Editor for Multiuser Communications for the IEEE TRANSACTIONS ON COMMUNICATIONS and as Associate Editor for Detection and Estimation for the IEEE TRANSACTIONS ON INFORMATION THEORY. He is General Co-Chair of ISITA 2012 and IEEE ISIT 2014. He received the EURASIP Journal of Wireless Communications and Networks (JWCN) Best Paper award in 2006. 